\title{
INDICADORES BIOQUIIMICOS Y PRUEBAS ISOMÉTRICAS EN FIBROMIALGIA
}

\section{BIOCHEMICAL MARKERS AND ISOMETRIC TESTS IN FIBROMYALGIA}

\author{
MANUEL LÓPEZ ESPINO(1), ADOLFO GOZALO PALOMARES( ${ }^{(2)}$, \\ JOSÉ CARLOS MINGOTE ADÁN ${ }^{(3)}$, CÉSAR BOROBIA FERNÁNDEZ ${ }^{(4)}$
}

(1) Psicólogo clínico en consulta privada.

(2) Médico- Bioquímico Hospital 12 de Octubre. Madrid. España.

(3) Psiquiatra Hospital 12 de Octubre. madrid. España.

(4) Profesor Titular Toxicología y legislación Sanitaria.

\section{RESUMEN}

El síndrome fibromiálgico (SFM) es una entidad clínica bien definida de tipo sistémico cuyos síntomas y signos exploratorios son crónicos y fluctuantes, observándose la interacción de diferentes factores de riesgo y los mecanismos periféricos y centrales, así como varios factores psicosociales asociados.

No se conocen completamente los mecanismos etiopatogénicos implicados del síndrome fibromiálgico. El diagnóstico es clínico y tras la exclusión de otras patologías somáticas habitualmente se tarda demasiado tiempo para diagnosticar y tratar a las personas afectadas.

Los estudios realizados se han orientado a analizar la existencia de lesiones musculares, alteraciones en el sistema inmunológico, anomalías psicológicas, problemas hormonales, trastornos del sueño, niveles bajos de algunas sustancias importantes en el sistema nervioso, pero en la actualidad no existe un test específico para poder realizar su diagnóstico preciso.

En este trabajo adaptamos dos instrumentos de evaluación diagnóstica que son los indicadores bioquímicos y la isometría muscular, biomarcadores objetivos de diagnóstico que confirmen el SFM. Como enfermedad psicosomático y no como trastorno somatoforme.

Nuestro estudio se planteó como un análisis prospectivo de un colectivo de 26 pacientes del género femenino con fibromialgia y una muestra poblacional de referencia que aceptaron ser sometidos a una serie de pruebas isométricas y a la obtención de muestras biológicas para medir un perfil bioquímico de estrés.

Palabras clave: Fibromialgia, marcadores biológicos de diagnóstico, rendimientos dinámicos isométricos.

(Med Segur Trab 2008; 54 (213):47-66)

\section{ABSTRACT}

Fibromyalgia is a well defined clinic entity which systemic symptoms and signs are chronic and fluctuating, in which we can see the interaction of different s etiopathogenic mechanisms, and associated psychosocial factors as well.

There are not completely known the implicated etiopatogenic mechanisms. The diagnostic process is clinic and it usually takes too much time to diagnose and to treat people affected.

In spite of numerous studies, but actually it does not exist any specific test for a precise differential diagnostic. In this study we adapt two evaluation instruments, which are biological markers and muscular, in order to confirm the SFM as psychosomatic illness, and not as somatoforme disorder.

Our study was planned as a prospective analysis of 26 female patients affected by fibromyalgia and a random sample that accepted being studied, in order to measure the biological profile of stress and isometric and dynamic performances ( Isostation B- 200) in fibromyalgic patiens and control group.

Key words: Fibromyalgia, biological markes of diagnostic, isometric and dynamic performances.

(Med Segur Trab 2008; 54 (213):47-66) 


\section{INTRODUCCIÓN}

El nombre de Fibromialgia deriva de: "FIBROS" = tejidos blandos del cuerpo, "MIOS" = músculos y "ALGIA" = dolor. Es decir, "Dolor músculoesquelético". A esta definición podemos añadir, "generalizado crónico y de causa desconocida".

La fibromialgia representa un problema de salud pública por su elevada prevalencia, morbilidad, hiperfrecuentación y consumo sanitario, así como incapacidad laboral. Se calcula que en España existen un $2,4 \%$ de personas adultas afectadas, que alcanzan a ser en torno a 700.000 casos, con un pico de prevalencia entre los 40 y 49 años, en su mayor parte mujeres (95-97\%). Se estima que estas pacientes constituyen entre el 10 y el $20 \%$ de casos atendidos en las consultas de Reumatología y en las Unidades del dolor.

En el año, 1990, el American College of Rheumatology (ACR), a través de un comité multicéntrico propuso unos criterios para definir, diagnosticar y clasificar el Síndrome de Fibromialgia, que se siguen aplicando en la actualidad y que se incluye en la ICD-10 como "M79-O Rheumatism, unspecified". (1)

Los criterios diagnósticos son los siguientes:

- Dolor difuso de carácter crónico y de más de tres meses de evolución.

- Sensibilidad dolorosa aumentada en 11 o más de los 18 puntos de localizaciones precisas. Los puntos sensibles típicos o "tender points"

- Ausencia de otra enfermedad sistémica que pudiera ser la causa del dolor.

En el año 1992, fue reconocida por la OMS como una entidad clínica diferenciada denominándose "síndrome de fibromialgia", dentro del apartado de los "reumatismos de partes blandas". Se trata de un síndrome somático multisintomático caracterizado por un estado doloroso generalizado no articular que afecta fundamentalmente a los músculos, con elevada sensibilidad al dolor en múltiples puntos predefinidos y asociado con fatiga persistente, sueño no reparador y rigidez o entumecimiento, predominantemente matutinos. Estos criterios diagnósticos quedaron recogidos en el Consensus Document on Fibromyalgia, 1993. También en el año 1994 fue reconocida por la Asociación Internacional para el Estudio del Dolor y clasificada con el código X33.X8a. $(2,3,4)$.
Actualmente se considera que fibromialgia y fatiga crónica son dos entidades clínicas diferentes pero estrechamente relacionadas, con una superposición de síntomas que llega a ser de hasta el 74\% de los mismos, ya que comparten algunos factores etiopatogénicos mediadores, genéticos y neurobiológicos. En la actualidad se reconoce que los factores genéticos condicionan el umbral de sensibilidad y la tolerancia al dolor, como se constata en la habitual agregación de diferentes patologías dolorosas entre las personas afectadas y sus familiares, siendo de un $20 \%$ los familiares de primer grado afectados por el SFM, lo que supone un riesgo ocho veces mayor que en población general. En experimentación animal se ha constatado que la sensibilidad al dolor, al igual que la sensibilidad analgésica a la morfina es un rasgo que tiene una alta heredabilidad mediada a través de varios mecanismos genéticos reguladores del sistema opióide endógeno.

La patogénesis multifactorial de la fibromialgia no está del todo clara, por eso es útil considerarla como una alteración en la modulación del dolor debida a una deficiente disponibilidad de varias aminas biógenas y neuropéptidos moduladores en el sistema nervioso central. Múltiples experiencias constatan que los pacientes con fibromialgia tienen un estado generalizado de amplificación del dolor con factores periféricos y centrales involucrados en estos pacientes a los que diagnostica de "Síndrome de disfunción neuroendocrina", como se esquematiza en el siguiente esquema propuesto por Yunnus (5):

Se han descrito casos de fibromialgia que comienzan después de procesos inespecíficos tales como una infección bacteriana o viral, un accidente de automóvil, la separación matrimonial o el divorcio, experiencias traumáticas, etc. Estos agentes desencadenantes no causan la enfermedad, sino que la precipitan o la agravan en una persona que tiene una vulnerabilidad neurobiológica latente en su respuesta de estrés frente a determinados estímulos o demandas adaptativas, según el modelo diatesisestrés. En otros casos, hasta en un 50\% de afectadas, la fibromialgia es secundaria a otras enfermedades médicas tales como artritis reumatoide y lupus eritematoso.

Todos los modelos teóricos sobre el dolor reconocen que se trata de un proceso psicobiológico complejo que incluye cuatro dimensiones: biológica, temporal, cognitiva y emocional. El dolor es una experiencia intrínsecamente estresante donde elementos de diversa naturaleza pueden desempeñar un 
MODELO DE SFM DE YUNNUS

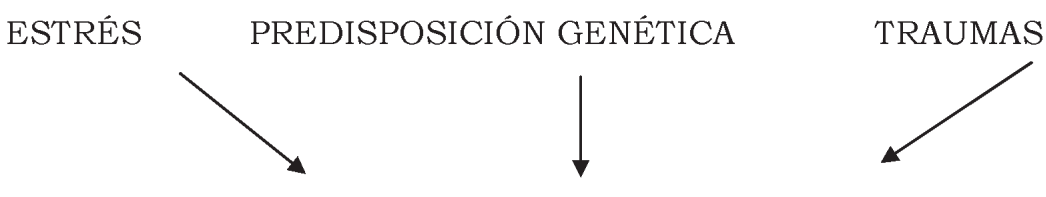

DISFUNCIÓN NEUROBIOLÓGICA HETEROGENEA

\section{MECANISMOS CENTRALES ABERRANTES}

DEL DOLOR Y LA FATIGA

ANSIEDAD
ESTRES
DEPRESIÓN
HIPERACTIVIDAD
SIMPÁTICA
HIPOOXIA
OTROS

SUEÑO NO REPARADOR

MIEDO AL DOLOR

INACTIVIDAD

MALA POSTURA

DESENTRENAMIENTO

Y TRAUMA FISICO

AMPLIFICACIÓN DEL DOLOR

FIBROMIALGIA

papel relevante en cada persona y en cada episodio padecido a lo largo del tiempo. No es de extrañar que las emociones asociadas al dolor sean las mismas que las relacionadas con el estrés, ya que el dolor crónico es un tipo particular de estrés.

\section{FACTORES ETIOPATOGÉNICOS}

Existe una fuerte evidencia en la actualidad de que los factores genéticos, así como la desregulación de varios neurotransmisores, y mediadores neuroendocrinos e inmunológicos son importantes mediadores del SFM. (5).

En los pacientes con fibromiálgia se han detectado diversas alteraciones en el sistema nervioso:

1. Niveles bajos de varias aminas biógenas en el líquido cefaloraquídeo (LCR), implicadas en la regulación del dolor, particularmente la serotonina, que es un neurotransmisor muy importante en la regulación central del dolor, sueño y humor, los tres parámetros más alterados en el SFM. También esta disminuido los niveles del L-tryptófano (precursor sanguíneo de la serotonina cerebral).
Las concentraciones de Noradrenalina y Dopamina en el LCR son mas bajas en los pacientes con fibromialgia que en los controles sanos. También se han demostrado valores bajos de Serotonina plasmática y concentraciones aumentadas de Sustancia P, que actúa como mediador de la nocicepción en el LCR de dichos pacientes. La Serotonina y Noradrenalina son sustancias mediadoras de la analgesia. La Serotonina también está involucrada en el inicio y perpetuación de la fase IV de sueño profundo NO REM, que se encuentra alterada en estos pacientes. El aumento de Sustancia P podría explicar un umbral anormalmente disminuido al dolor en esta enfermedad. También explicaría porqué los inhibidores de recaptación de Serotonina producen un cierto grado de beneficio en el tratamiento de la fibromialgia. (6, $7,8,9,10)$ La base fisiopatológica de este fenómeno es la activación de receptores de Nmetil-D-aspartato (NMDA). El sinergismo entre sustancia $\mathrm{P}$ y receptores NMIDA desempeña un papel fundamental en la perpetuación de la hiperalgesia secundaria. Sin embargo la fibromialgia es más que dolor muscular. Los rasgos multidimensionales de la fibromialgia pueden ser explicados por una respuesta 
variable mediada por los mecanismos de adaptación crónica al estrés.

2- Disfunción del eje hipotálamo-hipofiso-adrenal demostrado por la aparición en estos enfermos de una supresión de dexametasona anormal, niveles bajos de cortisol urinario libre, hiper respuesta de $\mathrm{ACTH}$ al CRF e hiporespuesta del cortisol frente a controles sanos. El hipocortisolismo que se encuentra también en otras enfermedades como el trastorno de estrés postraumático, se asocia a hiperalgesia y alodimia, con una percepción aumentada del dolor. (11).

3. Umbral del dolor disminuido debido a una anomalía funcional en el sistema nervioso central (SNC) asociada a un metabolismo y flujo sanguíneo regional cerebral más bajo del normal, asociado a varias alteraciones bioquímicas cerebrales: disminución de la dinorfina, una endorfina cerebral (lo cual explicaría la sensibilidad al dolor) y aumento de la sustancia $P$, junto con las alteraciones de los neurotransmisores ya señaladas.

4. Alteración del sueño, concretamente del sueño no REM. El sueño se divide esencialmente en sueño No REM (un sueño caracterizado por un umbral de vigilia relativamente alto y en el que no existe supresión del tono muscular) que se divide a su vez en fases 1-4 (siendo las dos últimas de ondas lentas características tanto teta como delta) y el sueño REM o de ondas rápidas, alfa, o sueño paradójico (de movimientos oculares rápidos caracterizado por un aumento del metabolismo cerebral, y es en el que se da la mayor actividad onírica). Moldofsky consiguió reproducir los signos y síntomas fibromiálgicos interrumpiendo con estímulos auditivos el sueño no REM de individuos control, no así cuando interrumpía el sueño REM, ya que en tal caso no conseguía reproducir este síndrome. Al hacer un EEG mientras dormían se comprobó que el sueño de las fases 3 y 4 , las ondas teta y delta de baja frecuencia, eran interrumpidas por otras ondas alfa de alta frecuencia, a lo que se denominó fenómeno de intrusión de ondas alfa, por lo que los pacientes con fibromialgia tienen un sueño fragmentado y de peor calidad.

Los estadios 3 y 4 del sueño se encuentran relacionados con la secreción de Hormona del Crecimiento, en ellas se produce entre el 50 y $80 \%$ de la producción total diaria de dicha hormona, en forma de picos nocturnos. El Eje GH-Somatomedina C se ha demostrado que es importante en los procesos de homeostasis y reparación muscular. Una disminu- ción de la Hormona del Crecimiento predispone al microtrauma muscular y retardaría la curación normal del músculo por un anabolismo reducido. Un estudio llevado a cabo en el Hospital Virgen del Rocío de Sevilla consistió en la medición de secreción continua de $\mathrm{GH}$ mediante bomba de extracción durante las horas nocturnas. Los resultados demostraron que los pacientes con fibromialgia presentaban una disminución de la secreción de GH que se tradujo en una disminución de los picos nocturnos estadísticamente significativos con respecto al grupo control. Se sabe que los adultos con disminución de esta hormona presentan disminución de la fuerza muscular. También se sabe que el tratamiento con la misma en ancianos revierte la pérdida de músculo. $(12,13,14,15,16,17,18)$.

5. Alteraciones inmunológicas. La Fibromialgia tiene síntomas en común con otra serie de enfermedades reumáticas que son de carácter autoinmune, como es la Artritis Reumatoide y el Lupus Eritematoso Sistémico. Hay diversos estudios en que los investigadores han identificado ciertos autoanticuerpos. Klein y colaboradores en un estudio publicado en 1992, encontraron anticuerpos antiserotonina en el $74 \%$ de los pacientes estudiados con fibromialgia, y en cambio no presentaban dichos anticuerpos los pacientes con otras enfermedades reumaticas como la artritis reumatoide, la polimialgia reumática o las enfermedades del colágeno. Estos resultados pueden explicar la disminución de los niveles plasmáticos de serotonina y de 5HIAA en LCR en estos enfermos en comparación con controles sanos, reflejo de una alteración de los mecanismos centrales del dolor, una disfunción neuroendocrino inmunológica compleja. En otro estudio llevado a cabo por Werle y colaboradores en el año 2001 también encontraron una alta cifra de anticuerpos antiserotonina en los pacientes fibromiálgicos comparados con los controles y además no encontraron diferencias en cuanto a edad o sexo. Tampoco encontraron relación de estos niveles con la sintomatología psicológica como depresión o ansiedad. $(19,20)$

Rodríguez Franco en 1995 ha encontrado una concentración plasmática disminuida de triptófano y un cociente de transporte del mismo disminuido en SFM frente a controles sanos.

6. Alteraciones musculoesqueléticas. Aarflot and Bruusgaard en 1996 encontraron una significativa correlación entre los niveles elevados de anticuerpos tiroideos microsomales y alteraciones musculoesqueléticas en una población de Noruega. Ocho pacientes que inicialmente presentaban síntomas de 
fibromialgia, tenían evidencias de hipotiroidismo. Los síntomas miálgicos se resolvieron en seis de ellos al ser tratados con hormona tiroidea a bajas dosis. En otros estudios se ha hipotetizado sobre la existencia de una disfunción neuroendocrina-inmune $(21,22)$.

Cuando en el año 1986, Lund y Bengtsson realizaron biopsias musculares y medidas de presión de oxígeno en músculos de pacientes afectados de fibromialgia, y considerando que la hipoxia muscular podía ser un factor patogénico importante en esta enfermedad, pensaron que igualmente podía existir otro factor igualmente importante a nivel del metabolismo intermediario. Para comprobar la posible alteración a este nivel, compararon las biopsias de músculos trapecios, en el punto de sensibilidad aumentada, de los pacientes con fibromialgia con los datos obtenidos de las biopsias de los controles sanos. Midieron los valores de ATP, ADP, AMP, fosfocreatina $(\mathrm{PCr})$, creatina, lactato, piruvato, glucógeno y la carga de energía potencial (CEP). Las concentraciones de ATP y ADP fueron significativamente más bajas en los trapecios de los pacientes con fibromialgia respecto a los controles y los valores de AMP estaban aumentados. El valor del pool de nucleótidos (ATP + ADP + AMP) también era más bajo y había un descenso significativo de la CEP. Los valores de $\mathrm{PCr}$ estaban significativamente más bajos y los de creatina, más altos. No había diferencias del contenido de lactato y piruvato respecto a los controles. (23)

Los niveles bajos, de la enzima fosfocreatina de las células musculares y de ATP, influyen en la regulación del flujo y reflujo de calcio en las células musculares, que es importante en la contracción y relajación del músculo. Si los niveles de ATP son bajos, el calcio no es "rechazado" y el músculo continua contraído, haciendo que se experimente y mantenga el dolor.

Respecto de los factores periféricos, es una evidencia que no existe defecto específico en el músculo de pacientes con SFM, aunque hay observaciones clínicas que señalan al músculo como origen del dolor. (24) la biopsia de los puntos gatillo, no revelan anomalías histológicas, aunque algunos autores destacan pequeñas diferencias al compararlas con la población control como son:

Bennett constató un menor flujo sanguíneo y una disminución del metabolismo del fosfato de alta energía en esas áreas y otros depósitos de inmunocomplejos dermoepidérmicos apareciendo positivi- dad a títulos bajos para los ANA, C3, IL-2, anormal actividad de los linfocitos NK, y depósito granular fino de la $\lg \mathrm{G}$, similar al lupus.(....)

Simms y col. observaron en los puntos gatillo un aumento del depósito amorfo intracelular con abundantes mastocitos plaquetas y miofilamentos intracelulares gigantes. (...)

Neeck y Riedel encontraron niveles sustancialmente bajos de calcio libre y niveles de parathormona más altos en pacientes con fibromialgia que en voluntarios normales. Además la calcitonina era significativamente más baja en estos pacientes. El origen de esta hipocalcemia es desconocido, aunque se piensa que una combinación de las alteraciones a nivel del Eje HPA y HPT podrían ser importantes en la fisiopatología del metabolismo del calcio. $(6,7$, $25,26,27)$

7. A nivel psicopatológico presentan trastornos cognitivos, del humor y de la memoria. La ansiedad y la depresión se presentan con igual frecuencia en la fibromialgia que en otras enfermedades dolorosas crónicas como pueden ser la lumbalgia o la artritis reumatoide. Por este motivo no se cree que sean fundamentales en la causa de la enfermedad, pero sí en el aumento de los síntomas. Mingote y Arigita han descrito el patrón de conducta Tipo $\mathrm{F}$ como un "complejo emoción-acción" característico de las pacientes fibromialgicas que viven inmersas en una lucha agotadora por lograr la superadaptación a un medio frustrante del que no se pueden distanciar, lo que las mantiene en un estado de generalizada activación. (28).

\section{DIFICULTADES DIAGNÓSTICAS}

El diagnóstico de la fibromialgia no es un proceso fácil, ya que se precisa descartar otra patología de tipo inflamativo y enfermedades sistémicas como el hipotiroidismo. Existe el riesgo de sobrediagnosticarla y pasar por alto otras enfermedades que tienen un tratamiento eficaz diferente, como de infradiagnosticarla sobre todo cuando hay otras enfermedades osteomusculares y la fibromialgia es concomitante o secundaria.

La Resonancia magnética es una técnica útil para el estudio del metabolismo oxidativo del músculo en reposo y en ejercicio. En un estudio donde se ha utilizado en vivo con 31P, Los resultados han sido controvertidos, aunque parece que no existen diferencias estadísticamente significativas entre pacientes fibromiálgicos y controles. (29) 
La isometría mide indirectamente y registra periódicamente un esfuerzo muscular específico del aparato locomotor realizado por un individuo de forma continua y sensiblemente creciente, en contra de una fuerza de resistencia estática hasta alcanzar una situación de equilibrio mecánico y valora la articulación sana y la articulación dañada con objeto de determinar el grado de afectación al comparar simétrica y bilateralmente dos articulaciones. Permite que cada una de estas articulaciones sean examinadas en posición neutra y en cualquier dirección. Sus resultados pueden determinar el término medio de fuerza de una articulación a través de su rango de movimiento. La isometría cuantifica y evalúa de forma precisa, objetiva, fiable y segura la capacidad física, funcionalidad e integridad articular y muscular real del aparato locomotor en el ser humano, tanto en condiciones normales como patológicas. Idoate García y col. han estudiado a las pacientes con fibromialgia a través de la realización de estudios isocinéticos apreciandose una menor eficiencia funcional frente a controles sanos.(30)

\section{ANÁLISIS DE LOS RESULTADOS DE INDICA- DORES BIOQUÍMICOS DE ESTRÉS Y PRUE- BAS ISOMÉTRICAS MEDIDOS EN UN GRUPO DE PACIENTES CON FIBROMIALGIA.}

\section{MATERIAL Y MÉTODO}

Los indicadores bioquímicos, que nos permitirán cualificar o cuantificar el estrés, son los utilizados para explorar las funciones simpática, serotoninérgica, tiroidea, adrenal y prolactinémica. Los parámetros para función simpática, que se midieron en orina de 24 horas, fueron catecolaminas libres totales (CF), adrenalina (A), noradrenalina (NA), dopamina (DA) y el ácido vanilmandélico (VMA). Para función serotoninérgica se midió el ácido 5-hidroxiindolacético (HIAA) en orina de 24 horas. La función tiroidea se valoró, en suero, con tirotropina (TSH), tiroxina total (T4T) y tiroxina libre (T4L). Para la corticoadrenal se determinó el cortisol sérico (CORT). La función prolactinémica nos la dió la prolactina sérica (PRL). En un grupo concreto se midió también beta-endorfina sérica ( $\beta E N D)$.Los valores de concentración obtenidos, para cada parámetro, se relativizaron según los correspondientes a una muestra poblacional de referencia de su propio sexo, estableciendo grados de disfunción. Así mismo, se relativizaron los valores con cocientes entre parámetros, para evidenciar predominio de actividad (PRL/TSH, CF/CORT, NA/A, IMA, HIAA/VMA). Conviene recordar y mantener las reservas manifestadas en el documento elaborado sobre la estimación preliminar de estos indicadores.

El test isométrico mide con precisión la capacidad de realizar un esfuerzo muscular. Los datos proporcionan una medición exacta necesaria para identificar las áreas débiles, cuantificar las posibles deficiencias, determinar la extensión de la lesión o de la incapacidad, y por último, evaluar los objetivos de la rehabilitación y el progreso obtenido.

Para conseguir la mayor validez y fiabilidad de los resultados cada test es evaluado por cálculo computerizado con un coeficiente de variación de $18 \%$ o menos que indica que el paciente ha realizado un esfuerzo consistente.

Este método emplea una silla especial de medición que proporciona un aislamiento de los grupos musculares de interés en cada test, con objeto de evitar el solapamiento de otros grupos para asegurar la validez y fiabilidad de los resultados.

Las pruebas isométricas realizadas permitieron valorar una serie de parámetros distribuidos en los siguientes capítulos:

- Movimiento rotacional externo (Movi ROM, o Range of Motion), por rotaciones, flexión-extensión (Flex-ext.) y flexión lateral (Flex-lat.).

- Capacidades Isométricas de rotación derecha (Rot.dcha.), rotación izquierda (Rot.izqd.), flexión (Flex.), extensión (Ext.), flexión lateral derecha (F.L.D.), flexión lateral izquierda (F.L.I.).

- Media de los parámetros de la función flexoextensora contemplando movimiento rotacional externo (ROM), torsión máxima (Tor. max), torsión media (Tor.med), velocidad máxima (Vel. max), velocidad media (Vel. med), Impulso, Trabajo y Potencia.

- Media de las Velocidades angulares máxima y media de los movimientos raquídeos a estudio con carga isotónica del $25 \%$ incluyendo rotación derecha-izquierda (Rot. der-izqda.), flexiónextensión (Flex. - Ext.) y flexión lateral izquierda derecha F.L.I.D.) midiendo su velocidad angular máxima (V. A. max) y media (V. A. med).

En la Tabla 1 englobamos todos los parámetros que hemos medido, diferenciando los aspectos bioquímicos e isométricos y con las abreviaturas con las que aparecerán indicadas en el texto y las tablas de resultados, con el fin de simplificar su elaboración y poder agrupar mayor número de parámetros que facilite su revisión en conjunto. 
Tabla 1. Parámetros incluidos en la exploración.

\begin{tabular}{|c|c|c|}
\hline \multicolumn{2}{|c|}{ Indicadores Bioguimicos } & \multirow{2}{*}{ Pruebas isométricas } \\
\hline Sangre & Orina de $24 \mathrm{~h}$ & \\
\hline $\begin{array}{l}\text { Función } \\
\text { tiroidea: }\end{array}$ & Función simpática: & $\begin{array}{l}\text { Movimiento rotacional externo (Movi ROM): } \\
\text { Rotaciones, flexión-extensión (Flex-ext.), flexiôn } \\
\text { lateral (Flex-lat.) }\end{array}$ \\
\hline Tirotropina & Ácido vanilmandélico & Capacidades Isométricas: \\
\hline (TSH) & (VMA) & Rotación derecha (Rot.dcha.), 1 \\
\hline $\begin{array}{l}\text { Tiroxina } \\
\text { libre (T4L) }\end{array}$ & $\begin{array}{l}\text { Catecolaminas libres } \\
\text { totales }(\mathbf{C F})\end{array}$ & (Rot.izqd.), flexion (Flex.), extension (Ext.), flexion \\
\hline \multirow{2}{*}{$\begin{array}{l}\text { Tiroxina } \\
\text { total (T4T) }\end{array}$} & Adrenalina $(\mathbf{A})$ & \\
\hline & Noradrenalina (NA) & $\begin{array}{l}\text { Media de los parámetros de la función flexo- } \\
\text { extensora: }\end{array}$ \\
\hline \multirow{2}{*}{$\begin{array}{l}\text { Función } \\
\text { corticoadre } \\
\text { nal: }\end{array}$} & Dopamina (DA) & 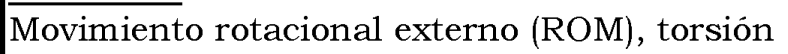 \\
\hline & $\begin{array}{l}\text { Indice metabólico } \\
\text { adrenérgico (IMA) }\end{array}$ & $\begin{array}{l}\text { máxima (Tor.max), torsión media (Tor.med), } \\
\text { velocidad máxima (Vel. max), velocidad media } \\
\text { (Vel. med), Impulso, Trabajo y Potencia }\end{array}$ \\
\hline $\begin{array}{l}\text { Cortisol } \\
\text { (COR) }\end{array}$ & \multirow{3}{*}{$\begin{array}{l}\text { Función } \\
\text { serotoninérgica: } \\
\text { Ácido 5- } \\
\text { hidroxiindolacético } \\
\text { (HIAA) }\end{array}$} & $\begin{array}{l}\text { Media de las Velocidades angulares máxima y } \\
\text { media de los movimientos raquídeos a estudio } \\
\text { con carga isotónica del } 25 \%:\end{array}$ \\
\hline $\begin{array}{l}\beta \text {-Endorfina } \\
\text { (ßEND) }\end{array}$ & & $\begin{array}{l}\text { Rotación derecha-izquierda (Rot. der-izqda.), } \\
\text { flexión-extensión (Flex. - Ext.) y flexión lateral } \\
\text { izquierda derech }\end{array}$ \\
\hline $\begin{array}{l}\text { Prolactina } \\
\text { (PRL) }\end{array}$ & & $\begin{array}{l}\text { velocidad angular máxima (V. A. max) y media } \\
\text { (V. A. med) }\end{array}$ \\
\hline
\end{tabular}

El grado de disfunción se valoró según el siguiente criterio:

0 al $5 \%$ de variación $=$ nivel conservado.

$5-10 \%$ de variación $=$ cambio ligero.

$10-15 \%$ de variación $=$ cambio discreto.

$15-30 \%$ de variación = cambio apreciable.

$30-40 \%$ de variación = cambio marcado.

Mas de $40 \%$ de variación = cambio importante.

\section{INDICADORES BIOQUÍMICOS DE ESTRÉS}

En primer lugar abordamos los indicadores bioquímicos de estrés analizando los resultados globalmente y distribuidos en dos grupos:

FMA: constituido por las pacientes orientadas a un tratamiento integral, consistente en un inhibidor selectivo de recaptación de serotonina, fisioterapia y psicoterapia.

FMB: constituido por las pacientes orientadas a un tratamiento exclusivamente con un inhibidor selectivo de recaptación de serotonina.

\section{Características de la muestra en su conjunto:}

Los resultados obtenidos en su conjunto y diferenciando las etapas pre y postratamiento aparecen en la tabla siguiente.

Los resultados obtenidos en su conjunto y diferenciando las etapas pre y postratamiento aparecen en la Tabla 2.

La muestra poblacional analizada tiene amplia dispersión, siendo máxima para PRL, TSH y $\beta E N D$, y mínima para T4L. El hecho de que la dispersión de la muestra sea grande está ligado a los parámetros medidos, que reflejan los distintos grados de disfunción en los que se deben encontrar los pacientes estudiados, resultando peculiar para el caso de $\beta E N D$ que se ha relacionado con el disestrés. La dispersión de prolactina, no obstante, la explicariamos por la existencia de individuos de distinta edad fértil, justificando la de TSH por distinto grado de hipofunción periférica por consumo . Llama la atención, sin embargo, la pequeña dispersión de T4L, que nos indicaría, que la homeostasis metabólica periférica se mantiene, independientemente de los cambios de 
Tabla 2. Análisis comparativo de indicadores bioqímicos de estrés.

\begin{tabular}{|c|c|c|c|c|c|c|c|c|c|c|c|c|c|c|c|c|c|c|}
\hline \multicolumn{19}{|c|}{ INDICADORES BIOQUÍMICOS DE ESTRÉS } \\
\hline & & & & \begin{tabular}{|c|}
$\mathbf{C F}$ \\
$\Phi$, Dia
\end{tabular} & $\begin{array}{l}\text { A } \\
\text { Dia }\end{array}$ & \begin{tabular}{|l|}
$\mathbf{A}$ \\
Dia
\end{tabular} & & & & $\begin{array}{l}\mathrm{SH} \\
\mathrm{d} / \mathrm{mL}\end{array}$ & $\begin{array}{c}T_{4} L \\
n g / d L\end{array}$ & $\begin{array}{c}\mathbf{T}_{\mathbf{T} T / \mathrm{T}} \\
\mu \mathrm{d} / \mathrm{dL}\end{array}$ & & & & A & $\begin{array}{l}\mathrm{CF} / \\
\mathrm{COR}\end{array}$ & \\
\hline \multicolumn{19}{|c|}{ muestra poblacional de referencia diferenciada por género } \\
\hline \multirow{3}{*}{\begin{tabular}{|l}
$\mathbf{g}$ \\
$\mathbf{F}$
\end{tabular}} & n & 50 & 68 & 68 & 68 & 68 & 68 & 50 & 50 & 65 & 65 & 65 & 10 & 50 & 68 & 68 & 50 & 45 \\
\hline & $\underline{x}$ & 2,30 & 3,70 & 43,7 & 5,4 & 38,2 & 220 & 16,0 & \begin{tabular}{|l|}
12,0 \\
\end{tabular} & 1,59 & 1,25 & 9,21 & 29.0 & 0,62 & \begin{tabular}{|l|}
95,6 \\
\end{tabular} & 10,9 & 2,73 & 7,54 \\
\hline & $\mathbf{c v} \%$ & 50 & 34 & 41 & 67 & 44 & 34 & 50 & 50 & 89 & 18 & 25 & 50 & 80 & 48 & 90 & 50 & 100 \\
\hline \multicolumn{19}{|c|}{ muestra poblacional con FM en su conjunto } \\
\hline \multirow{4}{*}{$\begin{array}{l}\mathrm{C} \\
\mathrm{O} \\
\mathrm{N} \\
\mathrm{J}\end{array}$} & n & 38 & 38 & 38 & 38 & 38 & 38 & 40 & 39 & 40 & 40 & 26 & 34 & 38 & 38 & 38 & 38 & 39 \\
\hline & $\underline{\mathbf{x}}$ & 28 & 3,95 & 32,5 & 4,2 & 27,7 & 202 & 13,7 & 12,9 & 2,18 & 1,14 & 7,76 & 14,5 & 1,28 & 140 & 7,44 & 2,60 & 9,82 \\
\hline & cv \% & 52 & 39 & 39 & 47 & 39 & 35 & 37 & 97 & 189 & 13 & 29 & 104 & 77 & 55 & 39 & 49 & 86 \\
\hline & $\begin{array}{l}\mathrm{FM} \\
\mathrm{frgF} \\
\end{array}$ & 1,86 & 1,06 & 0,74 & 84 & 0,73 &, 92 & 0,85 & 1,08 & 1,36 & 0,88 & 0,84 & 0,50 & 2,13 & 1,48 & 0,71 & 0,96 & 1,30 \\
\hline \multicolumn{19}{|c|}{ muestra poblacional con FM diferenciada por etapa terapeútica } \\
\hline \multirow{4}{*}{$\begin{array}{l}\mathbf{p} \\
\mathbf{r} \\
\mathbf{e}\end{array}$} & $n$ & 20 & 20 & 20 & 20 & 20 & 20 & 21 & 21 & 22 & 22 & 21 & 19 & 20 & 20 & 20 & 20 & 21 \\
\hline & $\mathbf{x}$ & 4,91 & \begin{tabular}{|l|}
4,21 \\
\end{tabular} & 32,5 & 4,2 & 28,2 & 216 & 13,5 & 10,7 & 2,81 & 1,15 & $\mathbf{7 , 7 4}$ & 18,8 & 1,36 & 146 & 8,02 & \begin{tabular}{|l|}
2,67 \\
\end{tabular} & 7,10 \\
\hline & cv \% & 55 & 37 & 31 & 54 & 32 & 32 & 35 & 81 & 194 & 15 & 30 & 82 & 76 & 56 & 39 & 46 & 94 \\
\hline & Pr/FM & 1,15 & 06 & 1,00 & 00 & 1,02 & 1,07 & 0,99 & 0,83 & 1,29 & 1,01 & 0,98 & 1,30 & 1,06 & 1,04 & 1,08 & 1,03 & 0,72 \\
\hline \multirow{4}{*}{$\begin{array}{l}\mathbf{p} \\
\mathbf{o} \\
\mathbf{s} \\
\mathbf{t}\end{array}$} & n & 18 & 8 & 18 & 8 & 18 & 18 & 19 & & 18 & 18 & 5 & 15 & 10 & 18 & 18 & 18 & 18 \\
\hline & $\mathbf{x}$ & 59 & 66 & 1,5 & 4,3 & \begin{tabular}{|l|}
27,2 \\
\end{tabular} & 186 & 13,8 & 15,5 & 1,40 & 1,14 & 7,86 & 9,0 & 1,20 & 134 & 6,80 & 2,53 & 13,0 \\
\hline & cv \% & 36 & 40 & 44 & 42 & 46 & 37 & 41 & 102 & 79 & 12 & 26 & 147 & 78 & 55 & 38 & 54 & 72 \\
\hline & t/FM & 84 & 93 & 97 & 02 & ,98 & 92 & 01 & 1,20 & 0,64 & 1,00 & 1,01 & 0,62 & 0,94 & 0,96 & 0,91 & 0,97 & 1,32 \\
\hline$\underline{\mathrm{p}}$ & $\leq$ & - & - & - & - & - & - & - & & - & 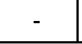 & - & 0,1 & 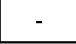 & - & 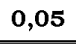 & - &, 05 \\
\hline \multicolumn{2}{|c|}{$\mathrm{p} / \mathbf{c o r}<$} & - & - & - & - & - & 05 & - & 05 & 0,05 & 0,01 & 0,1 & 0,05 & & - & 0,1 & - & 0,01 \\
\hline & & 11 & 12 & 74 & 84 & 74 & 1,00 & ,84 & 0,90 & \begin{tabular}{|l|}
1,65 \\
\end{tabular} & \begin{tabular}{|l|}
0,89 \\
\end{tabular} & 0,84 & 0,65 & 2,19 & 1,50 & 0,78 & 0,99 & 0,95 \\
\hline & $<$ & 01 & 5 & 01 & 5 & 01 & - & 05 & - & - & 5 & 0,05 & - & 5 &, 01 &, 0 & - & - \\
\hline \multicolumn{19}{|c|}{$\begin{array}{l}\text { Relación de los resultados obtenidos de la medición de indicadores bioquímicos de estrés y sus } \\
\text { cocientes en la muestra poblacional de referencia del género femenino (gF) y en la muestra poblacional } \\
\text { con sindrome de Fibromialgia (FM), en su conjunto (CONJ) y antes (pre) y después (post) del } \\
\text { tratamiento específico. Se describen el número de individuos (n), la media (x), el coeficiente de variación } \\
\text { en \% (cv \%), el cociente de los valores medios entre la muestra de pacientes FM y la muestra de } \\
\text { referencia (FM/rgF) y entre cada etapa terapéutica de la muestra con FM respecto a su conjunto (pr/FM } \\
\text { y pt/FM); indicándose además, en este último caso, el valor de p (p<) obtenido al comparar sus medias } \\
\text { con el test de la t de Student y la probabilidad de que sus resultados se ajusten a una correlación de } \\
\text { Pearson (p/cor<). En las dos últimas filas se expresa los resultados del cociente entre las medias } \\
\text { obtenidas de cada parámetro de las pacientes con FM en la etapa pretratamiento y los individuos de } \\
\text { referencia del género femenino (FM/rgF) y el valor de p al aplicar el test de la t de Student (p<). E1 } \\
\text { nombre completo de cada indicador y su abreviatura esta detallado en la Tabla } 1 .\end{array}$} \\
\hline
\end{tabular}

los otros sistemas neuroendocrinos implicados, lo que nos podría servir como un patrón interno al que referir los cambios que se operen.

$\mathrm{Su}$ valor central, o media, respecto a una población de referencia (sin estrés), presenta:

- Mayor predominio del sistema serotoninérgico (HIAA), que tiene un tono más aumentado que el simpático.

- Menor predominio noradrenérgico al estar más disminuida la NA que la A.

- Disminución del cortisol (CORT).
- Aumento de la prolactina (PRL) manteniendose la relación PRL/TSH.

- Mayor incremento de la TSH disminuyendo los niveles de T4L y T4T. Hay que considerar que hay un $12,5 \%$ de valores correspondientes a hipofunciones primarias tratadas y descompensadas.

- Los niveles de $\beta$-endorfina están marcadamente disminuidos.

Si buscamos interrelación entre los parámetros medidos encontramos las que figuran en la siguiente tabla, junto con las de la muestra poblacional de referencia: 
Indicadores bioquímicos y pruebas isométricas en fibromialgia Manuel López Espino, Adolfo Gozalo Palomares, José Carlos Mingote Adán, César Borobia Fernández

Cuadro 1. Interrelación entre indicadores bioquímicos de estrés.

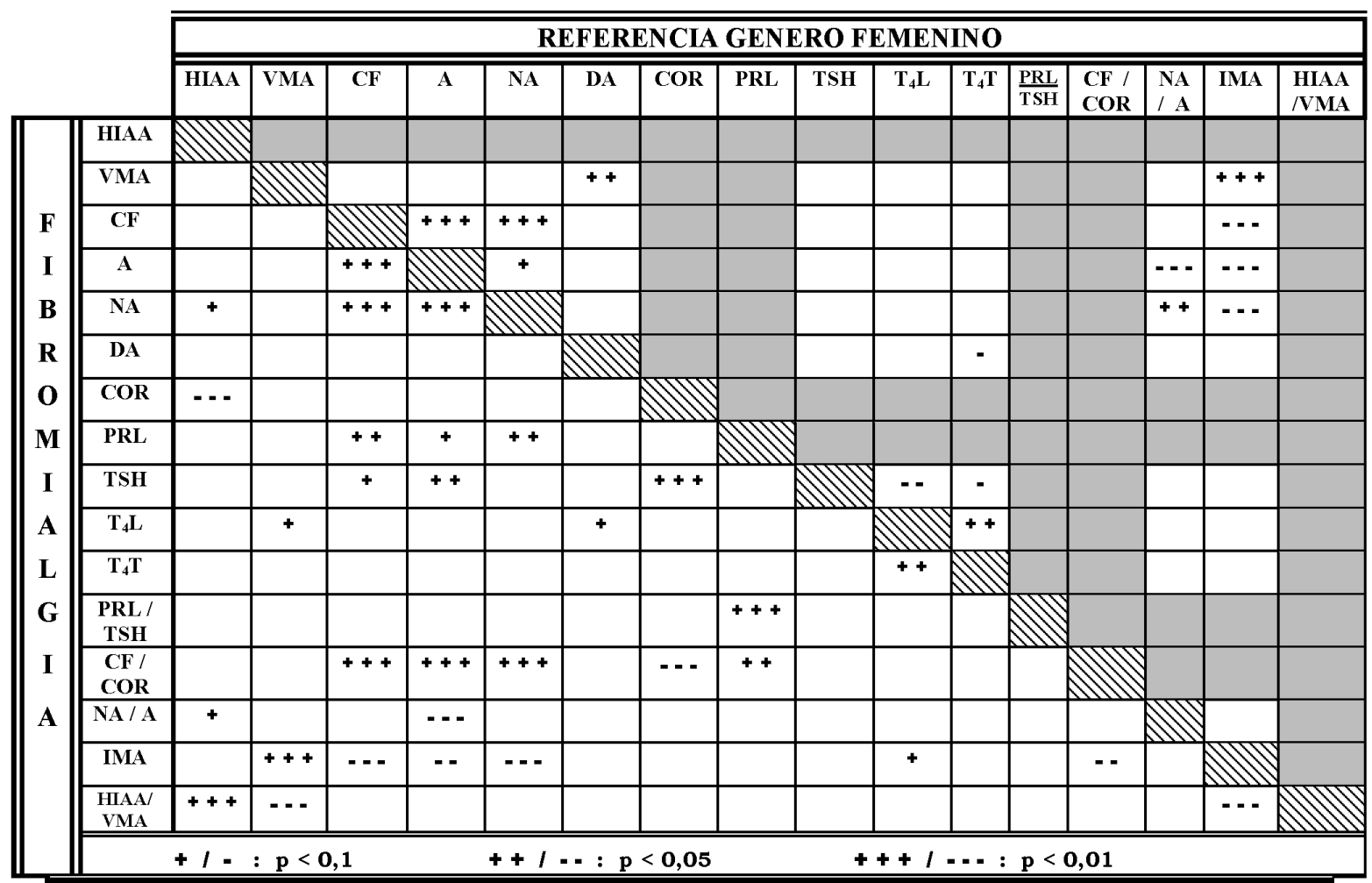

Presentación del análisis multiparamétrico sobre los resultados obtenidos con los indicadores

bioquímicos de estrés y sus cocientes buscando la existencia de correlación de Pearson en la muestra poblacional de referencia del género femenino y en la muestra poblacional con sindrome de Fibromialgia en su conjunto. Se describen en cada cuadrícula la probabilidad, con su signo, de que los resultados se ajusten considerando tres niveles : muy probable $(p<0,01)$, probable $(p<0,05)$, probabilidad incierta $(p$ $<0,1)$. E1 campo se ha dividido en dos áreas independientes mediante una diagonal de cuadrículas rayadas, para representar en la misma tabla los resultados de las sendas muestras poblacionales. Las cuadriculas sombreadas indican ausencia de datos. Las cuadriculas en blanco indican que la probalilidad de correlación era $>/=0,1$. El nombre completo de cada indicador y su abreviatura esta detallado en la Tabla 1.

Esta tabla merece unos comentarios adicionales que resalten las coincidencias o diferencias:

- En los pacientes se observa una marcada relación entre el indicador de la función simpatoadrenal (A) y el de la función simpatoperiférica (NA), relación que es débil en la muestra poblacional de referencia. Se aprecia, asímismo, una discreta asociación de NA con el indicador serotoninérgico (HIAA).

- La función tiroidea, de la población de referencia, refleja la situación homeostática con una estrecha relación inversa entre TSH y T4L y directa de T4L y T4T. Sin embargo, en los pacientes desaparece la relación TSH/T4L y surge la de TSH y A y una tendencia de asociación con NA.

- Otros aspectos, a destacar en los pacientes en su conjunto, incluirian: la relación inversa de HIAA y CORT; la relación directa del índice CF/CORT con $\mathrm{CF}$, A y NA e inversa con CORT; el índice NA/A pierde su relación con NA; el índice HIAA/VMA se correlaciona inversamente con VMA y directamente con HIAA.

Si desglosamos los resultados distribuidos según el tratamiento aplicado y etapa terapeútica obtenemos la tablas 3 . 
Tabla 3. Análisis de indicadores bioquímicos según terapéutica.

\begin{tabular}{|c|c|c|c|c|c|c|c|c|c|c|c|c|c|c|c|c|c|}
\hline \multicolumn{18}{|c|}{ INDICADORES BIOQUÍMICOS DE ESTRÉS } \\
\hline & $\begin{array}{l}\text { HIAA } \\
\text { mg Dia }\end{array}$ & \begin{tabular}{|l|l|l} 
VMA \\
mg.Dia
\end{tabular} & $\underset{\mu \text { 임ia }}{\text { CF }}$ & $\underset{\mu \mathrm{a} \text { Dia }}{\mathbf{A}}$ & $\underset{\mu \mathrm{o} / \text { Dia }}{\text { NA }}$ & $\underset{\mu \mathrm{g} / \text { Dia }}{\text { DA }}$ & $\begin{array}{c}\mathrm{COR} \\
\mu \mathrm{g} / \mathrm{dL}\end{array}$ & $\begin{array}{l}\text { PRL } \\
\mathbf{n g} / \mathrm{mL}\end{array}$ & $\begin{array}{c}\text { TSH } \\
\mu \mathrm{U} / \mathrm{mL}\end{array}$ & $\begin{array}{r}T_{4} \mathbf{L} \\
\mathrm{ng} / \mathrm{dL}\end{array}$ & $\begin{array}{r}\mathbf{T}_{4} \mathbf{T} \\
\mu \mathrm{g} / \mathrm{dL}\end{array}$ & $\begin{array}{c}\beta E N D \\
p(j / m L\end{array}$ & $\begin{array}{l}\text { HIAA } \\
\text { NMA }\end{array}$ & IMA & $\begin{array}{l}\text { NA } \\
\text { IA }\end{array}$ & $\begin{array}{c}\mathrm{CF} / \\
\mathrm{COR}\end{array}$ & $\begin{array}{l}\text { PRL/ } \\
\text { TSH }\end{array}$ \\
\hline \multicolumn{18}{|c|}{ muestra poblacional con FMA diferenciada por etapa terapeútica } \\
\hline $\mathbf{n}$ & 10 & 10 & 10 & 10 & 10 & 10 & 11 & 11 & 11 & 11 & 10 & 9 & 10 & 10 & 10 & 10 & 11 \\
\hline $\mathbf{r}$ & 5,13 & 4,61 & 32,6 & 4,7 & 28,0 & 187 & 13,1 & 13,7 & 1,64 & 1,13 & 7,86 & 15,3 & 1,45 & 162 & 7,45 & 2,86 & 9,41 \\
\hline \begin{tabular}{l|l} 
e & cv \% \\
\end{tabular} & 65 & 41 & 33 & 52 & 37 & 28 & 35 & 80 & 37 & 16 & 39 & 80 & 97 & 58 & 52 & 53 & 88 \\
\hline Pr/FM & 1,20 & 1,16 & 1,00 & 1,12 & 1,01 & 0,93 & 0,96 & 1,06 & 0,75 & 0,99 & 1,01 & 1,05 & 1,13 & 1,04 & 1,00 & 1,03 & 0,96 \\
\hline $\mathbf{p}$ & 14 & 14 & 14 & 14 & 14 & 14 & 15 & 14 & 14 & 14 & 4 & 11 & 14 & 14 & 14 & 14 & 14 \\
\hline o & 3,84 & 3,65 & 33,4 & 4,4 & 29,1 & 183 & 13,4 & 17,5 & 1,53 & 1,11 & 8,10 & 6,9 & 1,29 & 130 & 7,09 & 2,73 & 13,4 \\
\hline \begin{tabular}{l|l}
$\mathrm{s}$ & $\mathbf{c v} \%$
\end{tabular} & 35 & 37 & 45 & 42 & 47 & 35 & 39 & 100 & 80 & 11 & 28 & 97,1 & 80 & 60 & 38 & 52 & 74 \\
\hline \begin{tabular}{l|l}
$\mathbf{t}$ & $\mathrm{Pt} / \mathrm{FM}$ \\
\end{tabular} & 0,90 & 0,92 & 1,03 & 1,05 & 1,05 & 0,91 & 0,98 & 1,35 & 0,70 & 0,97 & 1,04 & 0,47 & 1,01 & 0,93 & 0,95 & 1,05 & 1,36 \\
\hline $\mathbf{p}<$ & - & 0,1 & - & - & - & - & - & 0,1 & - & - & - & & - & 0,05 & - & - & - \\
\hline $\mathrm{p} / \mathrm{cor}<$ & - & - & - & - & - & 0,01 & - & 0,1 & 0,05 & 0,01 & - & & - & - & 0,05 & - & 0,01 \\
\hline \multicolumn{18}{|c|}{ muestra poblacional con } \\
\hline n & 10 & 10 & 10 & 10 & 10 & 10 & 10 & 11 & 11 & 11 & 11 & 10 & 10 & 10 & 10 & 10 & 10 \\
\hline $\mathbf{r}$ & 4,69 & 3,82 & 32,5 & 3,7 & 28,5 & 246 & 13,9 & $\mathbf{7 , 4}$ & 3,97 & 1,16 & 7,63 & 22,1 & 1,26 & 129 & 8,60 & 2,47 & 4,57 \\
\hline \begin{tabular}{l|l} 
e & cv \% \\
\end{tabular} & 43 & 32 & 31 & $\mathbf{5 7}$ & 29 & 30 & 36 & 40 & 194 & 13 & 21 & 81 & 42 & 52 & 26 & 36 & 69 \\
\hline Pr/FM & 1,09 & 0,97 & 1,00 & 0,88 & 1,03 & 1,22 & 1,01 & 0,57 & $\mathbf{1 , 8 2}$ & 1,02 & 0,98 & 1,52 & 0,98 & 0,92 & 1,15 & 0,96 & 0,46 \\
\hline $\mathbf{p}$ & 4 & 4 & 4 & 4 & 4 & 4 & 4 & 4 & 4 & 4 & 1 & 4 & 4 & 4 & 4 & 4 & 4 \\
\hline o & 2,70 & 3,72 & 24,7 & 4,0 & 20,8 & 199 & 15,6 & 8,7 & 0,95 & 1,22 & 6,90 & 14,7 & 0,89 & 151 & 5,79 & 1,85 & 11,5 \\
\hline s $\quad$ cv \% & 18 & 56 & 27 & 46 & 28 & 50 & 50 & 31 & 41 & 15 & - & 170 & 52 & 44 & 39 & 53 & 71 \\
\hline $\begin{array}{lll} & \mathrm{Pt} / \mathrm{FM} \\
\end{array}$ & 0,63 & 0,94 & 0,76 & 0,95 & 0,75 & 1,14 & 1,14 & 0,67 & 0,43 & 1,07 & 0,89 & 1,01 & 0,69 & 1,08 & 0,78 & 0,71 & 1,17 \\
\hline $\mathbf{p}<$ & 0,05 & - & - & - & 0,1 & - & - & - & - & - & - & - & - & - & 0,05 & - & - \\
\hline p/cor $<$ & - & 0,01 & - & - & - & - & - & - & - & - & - & 0,05 & - & 0,05 & - & - & - \\
\hline
\end{tabular}

\begin{tabular}{|c|c|c|c|c|c|c|c|c|c|c|c|c|c|c|c|c|}
\hline \multicolumn{17}{|c|}{ diferencias entre FMA y FMB según la etapa terapeútica } \\
\hline p(pre) $<$ & - & - & - & - & - & 0,1 & - & 0,1 & - & - & - & - & - & - & - & 0,1 \\
\hline p(post) & 0,05 & - & 0,1 & - & 0,1 & - & - & 0,1 & 0,1 & - & - & - & - & - & - & - \\
\hline \multicolumn{17}{|c|}{$\begin{array}{l}\text { Relación de los resultados obtenidos de la medición de indicadores bioquímicos de estrés y sus cocientes } \\
\text { distribuidos en sendos grupos con sindrome de Fibromialgia (FM), sometidos a un tratamiento integral } \\
\text { (FMA) o a un tratamiento exclusivamente farmacológico (FMB), y antes (pre) y después (post) del } \\
\text { tratamiento especifico. Se describen el número de individuos (n), la media (x), el coeficiente de variación } \\
\text { en \% (cv \%), el cociente entre los valores medios de cada grupo, según el tratamiento aplicado y la etapa } \\
\text { terapéutica, y la muestra poblacional con FM en su conjunto (pr/FM y pt/FM); indicándose el valor de p } \\
\text { (p<) obtenido al buscar la efectividad de cada tratamiento con el test de la t de Student pareado y la } \\
\text { probabilidad de que sus resultados se ajusten a una correlación de Pearson (p/cor<). En las dos últimas } \\
\text { filas se expresa los resultados al confrontar con test de la te Student (p<) los datos obtenidos de FMA y } \\
\text { FMB en sus etapas pre o post tratamiento. El nombre completo de cada indicador y su abreviatura esta } \\
\text { detallado en la Tabla 1. }\end{array}$} \\
\hline
\end{tabular}

Del análisis de la Tabla 3 podemos destacar los siguientes comentarios:

- En la situación pre-tratamiento no se observan diferencias en los resultados obtenidos entre los dos grupos terapéuticos, al no tener implicaciones valorables las apuntadas para prolactina (PRL) y dopamina(DA).

- El grupo de pacientes con tratamiento integral revela cambios en su metabolismo adrenérgico, con disminución del ácido vanilmandélico y estabilización del valor de adrenalina, nivelándose con el de noradrenalina, y evidenciándose con el índice metabólico adrenérgico (IMA). El tono hipofisario, en sus funciones tiroidea y prolactinémica, guarda relación entre las etapas pre y post-tratamiento, así como el nivel de dopamina.

- En el grupo de pacientes con tratamiento exclusivamente farmacológico los mayores cambios los presenta el sistema serotoninérgico, con disminución de su actividad, disminución mayor que la presentada por el sistema simpático (fundamentalmente por la noradre- 
Indicadores bioquímicos y pruebas isométricas en fibromialgia Manuel López Espino, Adolfo Gozalo Palomares, José Carlos Mingote Adán, César Borobia Fernández

nalina) sobre el que tiene un predominio relativo (HIAA/VMA).

- Al interrelacionar los resultados obtenidos entre los dos grupos terapéuticos, en la situación post-tratamiento, se resaltan estas diferencias en los cambios funcionales de los indicadores bioquímicos de estrés, que se concretan en tres sistemas: sistema serotoninérgico con mayor disminución en el grupo FMB, sistema simpático con mejor estabilización funcional en el grupo FMA y la función tiroidea más óptimizada en el grupo FMA.

\section{PRUEBAS ISOMÉTRICAS}

Respecto a las pruebas isométricas se obtuvieron los siguientes resultados tabulados y distribuidos por capítulos según la exploración realizada:

\begin{tabular}{|c|c|c|c|c|c|c|c|c|c|}
\hline \multicolumn{10}{|c|}{ PARÁMETROS ISOMÉTRICOS (I) } \\
\hline & \multicolumn{3}{|c|}{ Movi ROM } & \multicolumn{6}{|c|}{ CAPACIDADES ISOMÉTRICAS (Nm) } \\
\hline & Rotaciones & Flex-ext. & Flex-lat. & Rot.dcha. & Rot.izqd. & Flex. & Ext. & F.L.D. & F.L.I. \\
\hline \multicolumn{10}{|c|}{ muestra poblacional con FM en su conjunto (fm) } \\
\hline $\mathbf{n}$ & 21 & 21 & 26 & 26 & 26 & 26 & 26 & 26 & 26 \\
\hline$x$ & 72,21 & 78,60 & 57,74 & 52,72 & 44,60 & 55,68 & 41,71 & 51,31 & 42,46 \\
\hline cv $\%$ & 25 & 32 & 23 & 45 & 58 & 45 & 47 & 43 & 45 \\
\hline
\end{tabular}

\begin{tabular}{|c|c|c|c|c|c|c|c|c|c|}
\hline \multicolumn{10}{|c|}{ muestra poblacional de referencia $(r)$} \\
\hline $\mathbf{n}$ & 10 & 10 & 10 & 10 & 10 & 10 & 10 & 10 & 10 \\
\hline $\mathbf{x}$ & 76,89 & 110,33 & 74,47 & 68,08 & 55,75 & 66,35 & 73,90 & 64,48 & 58,74 \\
\hline cv \% & 20 & 7 & 14 & 27 & 28 & 34 & 44 & 34 & 37 \\
\hline $\mathbf{f m} / \mathbf{r}$ & 0,94 & 0,71 & 0,77 & 0,77 & 0,80 & 0,84 & 0.56 & 0,79 & 0.72 \\
\hline$p<$ & - & 0,01 & 0,01 & 0,1 & 0,1 & - & 0,05 & 0,1 & 0,05 \\
\hline
\end{tabular}

\begin{tabular}{|c|c|c|c|c|c|c|c|}
\hline \multicolumn{8}{|c|}{ PARÁMETROS ISOMÉTRICOS (II) } \\
\hline \multicolumn{8}{|c|}{ MEDIA DE LOS PARÁMETROS DE LA FUNCIÓN FLEXO-EXTENSORA } \\
\hline $\begin{array}{l}\text { ROM } \\
\mathrm{g}\end{array}$ & $\begin{array}{l}\text { Torque max } \\
\text { Nm }\end{array}$ & \begin{tabular}{|l|} 
Torque med \\
Nm
\end{tabular} & $\begin{array}{l}\text { Vel. max } \\
\mathrm{g} / \mathrm{s}\end{array}$ & $\begin{array}{l}\text { Vel. med } \\
\mathrm{g} / \mathrm{s}\end{array}$ & $\begin{array}{l}\text { Impulso } \\
\text { Nm/s }\end{array}$ & $\begin{array}{l}\text { Trabajo } \\
\text { Nm }\end{array}$ & $\begin{array}{l}\text { Potencia } \\
\text { Nm }\end{array}$ \\
\hline
\end{tabular}

\section{muestra poblacional con FM en su conjunto (fm)}

\begin{tabular}{|l|l|l|l|l|l|l|l|l|}
\hline $\mathrm{n}$ & 14 & 26 & 26 & 26 & 26 & 26 & 26 & 26 \\
\hline $\mathrm{x}$ & 79,73 & 24,37 & 17,93 & 71,83 & 41,19 & 53,74 & 61,62 & 22,47 \\
\hline $\mathrm{cv} \%$ & 22 & 37 & 50 & 33 & 35 & 63 & 63 & 57 \\
\hline \hline
\end{tabular}

muestra poblacional de referencia $(r)$

\begin{tabular}{|l|l|l|l|l|l|l|l|l|}
\hline $\mathrm{n}$ & 10 & 10 & 10 & 10 & 10 & 10 & 10 & 10 \\
\hline $\mathrm{x}$ & 92,78 & 30,58 & 24,62 & 93,44 & 58,20 & 63,68 & 77,09 & 43,22 \\
\hline cv \% & 20 & 31 & 31 & 25 & 30 & 64 & 33 & 48 \\
\hline
\end{tabular}

\begin{tabular}{|l|l|l|l|l|l|l|l|l|}
\hline \hline $\mathrm{fm} / \mathbf{r}$ & 0,85 & 0,80 & 0,73 & 0,77 & 0,70 & 0,84 & 0,80 & 0,52 \\
\hline$p<$ & 0,1 & 0,1 & 0,05 & 0,05 & 0,05 & - & 0,1 & 0,05 \\
\hline & & & & & & & & \\
\hline
\end{tabular}




\begin{tabular}{|c|c|c|c|c|c|c|}
\hline \multicolumn{7}{|c|}{ PARÁMETROS ISOMÉTRICOS (III) } \\
\hline & \multicolumn{6}{|c|}{$\begin{array}{l}\text { MEDIA DE VELOCIDADES ANGULARES MAXIMA Y } \\
\text { RAQUÍDEOS A ESTUDIO CON CARGA ISOTÓNICA DEL } 25 \%\end{array}$} \\
\hline & \multicolumn{2}{|c|}{ Rot. der-izqda. } & \multicolumn{2}{|c|}{ Flex. - Ext. } & \multicolumn{2}{|l|}{ F.L.I.D. } \\
\hline & $\begin{array}{l}\text { V. A. max } \\
\mathrm{g} / \mathrm{s}\end{array}$ & $\begin{array}{l}\text { V. A. med } \\
\mathrm{g} / \mathrm{s}\end{array}$ & $\begin{array}{l}\text { V. A. max } \\
\text { g/s }\end{array}$ & $\begin{array}{l}\text { V. A. med } \\
g / s\end{array}$ & $\begin{array}{l}\text { V. A. max } \\
\text { g/s }\end{array}$ & $\begin{array}{l}\text { V. A. med } \\
\mathrm{g} / \mathrm{s}\end{array}$ \\
\hline \multicolumn{7}{|c|}{ muestra poblacional con FM en su conjunto(fm) } \\
\hline $\mathbf{n}$ & 17 & 18 & 17 & 18 & 17 & 18 \\
\hline $\mathbf{x}$ & 74,51 & 45,62 & 78,97 & 40,96 & 79,01 & 41,41 \\
\hline cv \% & 47 & 46 & 32 & 41 & 32 & 36 \\
\hline
\end{tabular}

\begin{tabular}{l}
\hline muestra poblacional de referencia $(\boldsymbol{r})$ \\
\begin{tabular}{|l|l|l|l|l|l|l|}
\hline n & 10 & 10 & 10 & 10 & 10 & 10 \\
\hline x & 82,12 & 59,30 & 90,20 & 52,64 & 99,64 & 61,75 \\
\hline cv $\%$ & 32 & 24 & 32 & 27 & 24 & 22 \\
\hline
\end{tabular}
\end{tabular}

\begin{tabular}{|l|l|l|l|l|l|l|}
\hline \hline $\mathbf{f m} / \mathbf{r}$ & 0,91 & 0,77 & 0,87 & 0,78 & 0,79 & 0,67 \\
\hline $\mathrm{p}<$ & - & 0,05 & - & 0,1 & 0,05 & 0,01 \\
\hline & & & & & & \\
\hline \hline
\end{tabular}

Relación de los resultados obtenidos con las pruebas isométricas en la muestra poblacional de referencia del género femenino ( $\mathrm{r}$ ) y en la muestra poblacional con síndrome de Fibromialgia en su conjunto (fm). Se describen el número de individuos (n), la media (x), el coeficiente de variación en \% (cv \%), el cociente de los valores medios entre la muestra de pacientes con sindrome de Fibromialgia y la muestra de referencia $(\mathrm{fm} / \mathrm{r})$; indicándose además, en este último caso, el valor de $\mathrm{p}(\mathrm{p}<)$ obtenido al comparar sus medias con el test de la t de Student. E1 nombre completo de cada prueba y su abreviatura esta detallado en la Tabla 1.

Se observa, al analizar estos datos, que su dispersión es heterogénea entre los distintos parámetros aunque siempre es menor en el grupo de individuos de referencia, con alguna excepción como la del impulso en la función flexo-extensora. Además al comparar las medias de los valores de los parámetros entre la muestra poblacional de referencia y los pacientes con fibromialgia se constatan las siguientes diferencias según su capítulo:

\section{Movi ROM:}

Difieren muy significativamente:

- Flex-ext.: los pacientes con FM presentan un valor un $29 \%$ mas bajo que los individuos de referencia.

- Flex-lat.: los pacientes con FM presentan un valor un $23 \%$ mas bajo que los individuos de referencia.

\section{Capacidades isométricas:}

Difieren significativamente:

- Ext.: los pacientes con FM presentan un valor un $44 \%$ mas bajo que los individuos de referencia.

- F.L.I.: los pacientes con FM presentan un valor un $28 \%$ mas bajo que los individuos de referencia.

Difieren casi significativamente:

- Rot.dcha.: los pacientes con FM presentan un valor un $23 \%$ mas bajo que los individuos de referencia.

- Rot.izqd.: los pacientes con FM presentan un valor un $20 \%$ mas bajo que los individuos de referencia.

- F.L.D.: los pacientes con FM presentan un valor un $20 \%$ mas bajo que los individuos de referencia. 
Indicadores bioquímicos y pruebas isométricas en fibromialgia Manuel López Espino, Adolfo Gozalo Palomares, José Carlos Mingote Adán, César Borobia Fernández

\section{Media de los parámetros de la función flexo-exten- sora:}

Difieren significativamente:

- Tor.med.: los pacientes con FM presentan un valor un $27 \%$ mas bajo que los individuos de referencia.

- Vel.max: los pacientes con FM presentan un valor un $23 \%$ mas bajo que los individuos de referencia.

- Vel.med: los pacientes con FM presentan un valor un $30 \%$ mas bajo que los individuos de referencia.

- Potencia: los pacientes con FM presentan un valor un $28 \%$ mas bajo que los individuos de referencia.

Difieren casi significativamente:

- ROM.: los pacientes con FM presentan un valor un $15 \%$ mas bajo que los individuos de referencia.

- Tor.max: los pacientes con FM presentan un valor un $20 \%$ mas bajo que los individuos de referencia.

- Trabajo: los pacientes con FM presentan un valor un $20 \%$ mas bajo que los individuos de referencia.
Media de velocidades angulares máxima y media de los movimientos raquídeos a estudio con carga isotónica del $25 \%$ :

Difieren muy significativamente:

- V.A.med/F.L.I.D.: los pacientes con FM presentan un valor un $33 \%$ mas bajo que los individuos de referencia.

Difieren significativamente:

- V.A.med./Rot.der-izqda.: los pacientes con FM presentan un valor un $23 \%$ mas bajo que los individuos de referencia.

- V.A.max/F.L.I.D.: los pacientes con FM presentan un valor un $21 \%$ mas bajo que los individuos de referencia.

Difieren casi significativamente:

- V.A.med/Flex-Ext: los pacientes con FM presentan un valor un $22 \%$ mas bajo que los individuos de referencia.

Si buscamos interrelación entre las distintas pruebas isométricas encontramos las que figuran en la siguiente tabla:

\begin{tabular}{|c|c|c|c|c|c|c|c|c|c|c|c|}
\hline & & & \multicolumn{9}{|c|}{ REFERENCIA } \\
\hline & & & \multicolumn{3}{|c|}{ Movi ROM } & \multicolumn{6}{|c|}{ CAPACIDADES ISOMÉTRICAS (Nm) } \\
\hline & & & Rotaciones & Flex-ext. & Flex-lat. & Rot.dcha. & Rot.izqd. & Flex. & Ext. & F.L.D. & F.L.I. \\
\hline \multirow{10}{*}{ FM } & \multirow{3}{*}{$\begin{array}{l}\text { Movi } \\
\text { ROM }\end{array}$} & Rotaciones & & & & & & & & & \\
\hline & & Flex-ext. & +++ & & ++ & & & + & + & + & \\
\hline & & Flex-lat. & +++ & +++ & & & & & + & + & ++ \\
\hline & \multirow{6}{*}{$\begin{array}{l}\text { CAP. } \\
\text { ISOM } \\
(\mathrm{Nm})\end{array}$} & Rot.dcha. & & & & & + & & & & \\
\hline & & Rot.izqd. & + & ++ & + & +++ & & + & + & + & + \\
\hline & & Flex. & +++ & +++ & +++ & +++ & +++ & & +++ & +++ & +++ \\
\hline & & Ext. & +++ & ++ & +++ & +++ & +++ & +++ & & +++ & +++ \\
\hline & & F.L.D. & + & ++ & ++ & +++ & +++ & +++ & +++ & & +++ \\
\hline & & F.L.I. & +++ & +++ & +++ & +++ & +++ & +++ & +++ & +++ & \\
\hline & $1=$ & $p<0,1$ & & $I=$ & $<0,05$ & & ++1 & $\because p$ & & & \\
\hline
\end{tabular}




\begin{tabular}{|c|c|c|c|c|c|c|c|c|c|c|}
\hline & & \multicolumn{9}{|c|}{ REFERENCIA } \\
\hline & & \multicolumn{3}{|c|}{ Movi ROM } & \multicolumn{6}{|c|}{ CAPACIDADES ISOMÉTRICAS (Nm) } \\
\hline & & Rotaciones & Flex-ext. & Flex-lat. & Rot.dcha. & Rot.izqd. & Flex. & Ext. & F.L.D. & F.L.I. \\
\hline \multirow{8}{*}{$\begin{array}{l}\text { Media } \\
\text { de los } \\
\text { pará- } \\
\text { metros } \\
\text { de la } \\
\text { función } \\
\text { flexoex- } \\
\text { tensora }\end{array}$} & ROM $g$ & & + & + & & & & & & \\
\hline & Tor.max Nm & +++ & + & & & & & & & \\
\hline & Tor.med Nm & +++ & + & & & & & & & \\
\hline & Vel.max $\mathrm{g} / \mathrm{s}$ & & & & & & & & & \\
\hline & Vel.med $\mathrm{g} / \mathrm{s}$ & & & & & & & & & \\
\hline & Impulso $\mathrm{Nm} / \mathrm{s}$ & & & & & & & & + & + \\
\hline & Trabajo Nm & & & & & & & & & \\
\hline & Potencia Nm & & & + & & & & & & \\
\hline
\end{tabular}

\begin{tabular}{|c|c|c|c|c|c|c|c|c|c|c|}
\hline & & \multicolumn{9}{|l|}{ FM } \\
\hline & & \multicolumn{3}{|c|}{ Movi ROM } & \multicolumn{6}{|c|}{ CAPACIDADES ISOMÉTRICAS (Nm) } \\
\hline & & Rotaciones & Flex-ext. & Flex-lat. & Rot.dcha. & Rot.izqd. & Flex. & Ext. & F.L.D. & F.L.I. \\
\hline \multirow{8}{*}{$\begin{array}{l}\text { Media } \\
\text { de los } \\
\text { pará- } \\
\text { metros } \\
\text { de la } \\
\text { función } \\
\text { flexoex- } \\
\text { tensora }\end{array}$} & ROM $_{\mathbf{g}}$ & ++ & +++ & ++ & & & & & + & ++ \\
\hline & Tor.max Nm & & & & ++ & ++ & +++ & ++ & +++ & +++ \\
\hline & Tor.med Nm & & & & ++ & ++ & + & + & +++ & ++ \\
\hline & Vel.max $\mathrm{g} / \mathrm{s}$ & + & +++ & ++ & & & ++ & & + & \\
\hline & Vel.med $\mathrm{g} / \mathrm{s}$ & & +++ & + & & + & ++ & & ++ & ++ \\
\hline & Impulso $\mathrm{Nm} / \mathrm{s}$ & & & & + & + & & & & \\
\hline & Trabajo Nm & & + & & ++ & ++ & +++ & ++ & +++ & +++ \\
\hline & \begin{tabular}{|l|} 
Potencia Nm \\
\end{tabular} & & ++ & + & + & + & +++ & +++ & +++ & +++ \\
\hline
\end{tabular}

\begin{tabular}{|c|c|c|c|c|c|c|c|c|c|c|c|}
\hline & & & \multicolumn{9}{|c|}{ REFERENCIA } \\
\hline & & & \multicolumn{3}{|c|}{ Movi ROM } & \multicolumn{6}{|c|}{ CAPACIDADES ISOMÉTRICAS (Nm) } \\
\hline & & & Rotaciones & Flex-ext. & Flex-lat. & Rot.dcha. & Rot.izqd. & Flex. & Ext. & F.L.D. & F.L.I. \\
\hline \multirow{6}{*}{$\begin{array}{l}\text { Media de } \\
\text { velocida- } \\
\text { des angu- } \\
\text { lares } \\
\text { máxima y } \\
\text { media de } \\
\text { los movi- } \\
\text { mientos } \\
\text { raquídeos } \\
\text { a estudio. } \\
\text { Carga } \\
\text { isotón. del } \\
25 \%\end{array}$} & \multirow{2}{*}{$\begin{array}{l}\text { Rot. } \\
\text { der- } \\
\text { izqd } \\
\text { a. }\end{array}$} & \begin{tabular}{|l} 
V. A.max \\
g/s \\
\end{tabular} & & & & & & & & & \\
\hline & & \begin{tabular}{|l|} 
V. A.med \\
g/s
\end{tabular} & & & & & & & & & \\
\hline & \multirow{2}{*}{$\begin{array}{l}\text { Flex. } \\
- \\
\text { Ext. }\end{array}$} & \begin{tabular}{|l} 
v. A.max \\
g/s
\end{tabular} & & & ++ & & & & & & \\
\hline & & \begin{tabular}{|l} 
V. A.med \\
g/s
\end{tabular} & & & & & & & & & + \\
\hline & \multirow{2}{*}{$\begin{array}{l}\text { F.L.I } \\
\text { D. }\end{array}$} & \begin{tabular}{|l} 
V. A.max \\
g/s
\end{tabular} & & & & & & & & & \\
\hline & & $\begin{array}{l}\text { V. A.med } \\
\text { g/s }\end{array}$ & & & ++ & & & + & & & \\
\hline
\end{tabular}


Indicadores bioquímicos y pruebas isométricas en fibromialgia Manuel López Espino, Adolfo Gozalo Palomares, José Carlos Mingote Adán, César Borobia Fernández

\begin{tabular}{|c|c|c|c|c|c|c|c|c|c|c|c|}
\hline & & & \multicolumn{9}{|l|}{ FM } \\
\hline & & & \multicolumn{3}{|c|}{ Movi ROM } & \multicolumn{6}{|c|}{ CAPACIDADES ISOMÉTRICAS (Nm) } \\
\hline & & & Rotaciones & Flex-ext. & Flex-lat. & Rot.dcha. & Rot.izqd. & Flex. & Ext. & F.L.D. & F.L.I. \\
\hline \multirow{6}{*}{\begin{tabular}{|l} 
Media de \\
velocida- \\
des angu- \\
lares \\
máxima y \\
media de \\
los movi- \\
mientos \\
raquideos \\
a estudio. \\
Carga \\
isotón. del \\
$25 \%$
\end{tabular}} & \multirow{2}{*}{\begin{tabular}{|l|} 
Rot. \\
der- \\
izqd \\
a. \\
\end{tabular}} & $\begin{array}{l}\text { V. A.max } \\
\text { g/s }\end{array}$ & & & & & + & & & & \\
\hline & & \begin{tabular}{|l}
$\begin{array}{l}\text { V. A.med } \\
\text { g/s }\end{array}$ \\
\end{tabular} & & ++ & & + & ++ & +++ & & ++ & \\
\hline & \multirow{2}{*}{\begin{tabular}{|l} 
Flex. \\
- \\
Ext.
\end{tabular}} & $\begin{array}{l}\text { V. A.max } \\
\text { g/s }\end{array}$ & & +++ & + & & ++ & + & & ++ & \\
\hline & & \begin{tabular}{|l} 
V. A.med \\
g/s
\end{tabular} & & +++ & & & ++ & ++ & & +++ & ++ \\
\hline & \multirow{2}{*}{$\begin{array}{l}\text { F.L.I } \\
\text {.D. }\end{array}$} & \begin{tabular}{|l|}
$\begin{array}{l}\text { V. A.max } \\
\text { g/s }\end{array}$ \\
\end{tabular} & & + & & & ++ & + & & + & \\
\hline & & $\begin{array}{l}\text { v. A.med } \\
\text { g/s }\end{array}$ & & ++ & & & +++ & + & & ++ & \\
\hline
\end{tabular}

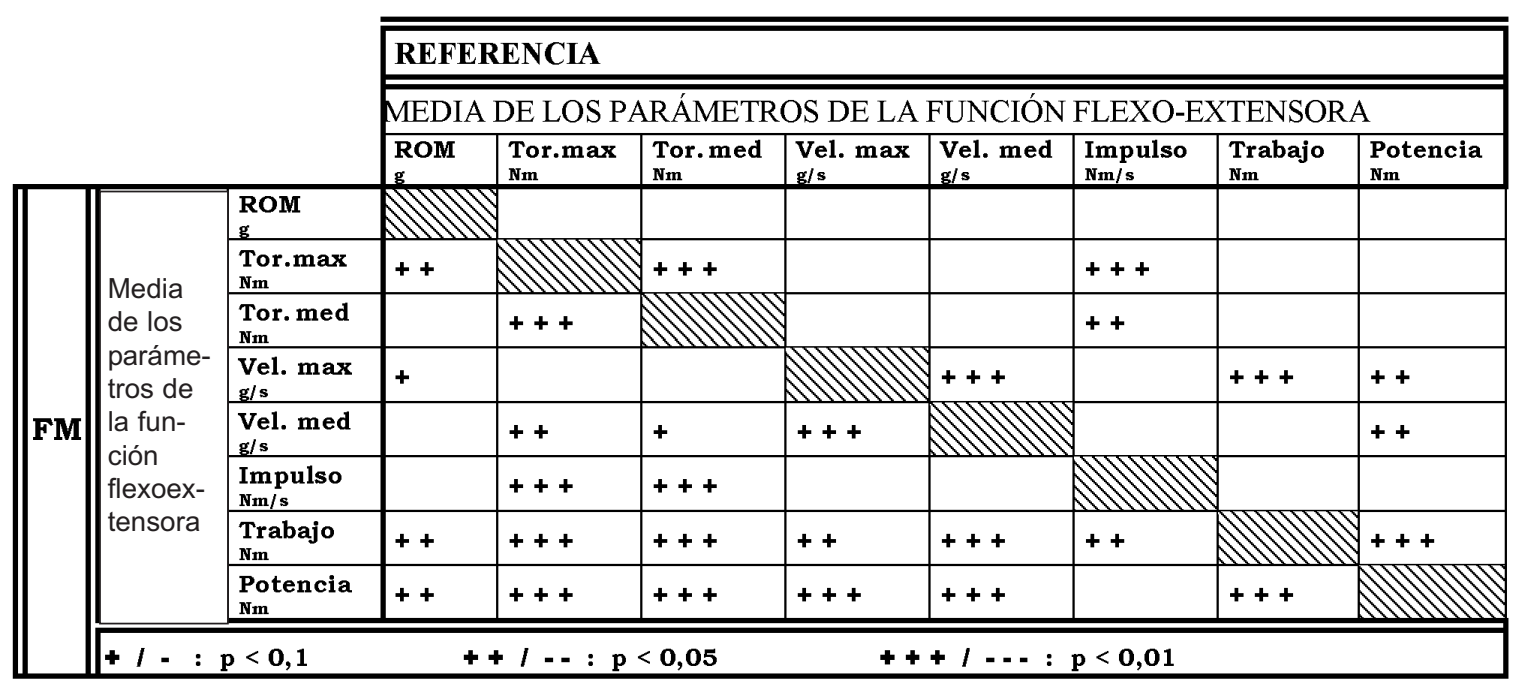

\begin{tabular}{|c|c|c|c|c|c|c|c|c|c|c|}
\hline & & & \multicolumn{8}{|c|}{ REFERENCIA } \\
\hline & & & \multicolumn{8}{|c|}{ MEDIA DE LOS PARÁMETROS DE LA FUNCIÓN FLEXO-EXTENSORA } \\
\hline & & & \begin{tabular}{|l} 
ROM \\
$\mathrm{g}$
\end{tabular} & \begin{tabular}{|l|} 
Tor.max \\
Nm
\end{tabular} & $\begin{array}{l}\text { Tor. med } \\
\text { Nm }\end{array}$ & $\begin{array}{l}\text { Vel. } \max \\
\mathrm{g} / \mathrm{s}\end{array}$ & $\begin{array}{l}\text { Vel. med } \\
\mathrm{g} / \mathrm{s}\end{array}$ & $\begin{array}{l}\text { Impulso } \\
\text { Nm/s }\end{array}$ & \begin{tabular}{|l} 
Trabajo \\
Nm
\end{tabular} & $\begin{array}{l}\text { Potencia } \\
\text { Nm }\end{array}$ \\
\hline \multirow{6}{*}{$\begin{array}{l}\text { Media de } \\
\text { velocida- } \\
\text { des angu- } \\
\text { lares } \\
\text { máxima y } \\
\text { media de } \\
\text { los movi- } \\
\text { mientos } \\
\text { raquideos } \\
\text { a estudio. } \\
\text { Carga } \\
\text { isotón. del } \\
25 \%\end{array}$} & \multirow{2}{*}{\begin{tabular}{|l|} 
Rot. \\
der- \\
izqd \\
a.
\end{tabular}} & $\begin{array}{l}\text { V. A.max } \\
\text { g/s }\end{array}$ & & & & ++ & + & & & \\
\hline & & \begin{tabular}{|l|} 
V. A.med \\
g/s
\end{tabular} & & & & + & ++ & & & \\
\hline & \multirow{2}{*}{\begin{tabular}{|l} 
Flex. \\
- \\
Ext.
\end{tabular}} & \begin{tabular}{|l|} 
V. A.max \\
g/s
\end{tabular} & & & & +++ & +++ & & ++ & +++ \\
\hline & & $\begin{array}{l}\text { v. A.med } \\
\text { g/s } \\
\end{array}$ & & & & & & & + & \\
\hline & \multirow{2}{*}{$\begin{array}{l}\text { F.L.I } \\
\text { D. }\end{array}$} & \begin{tabular}{|l|} 
V. A.max \\
g/s \\
\end{tabular} & & & & ++ & ++ & & ++ & +++ \\
\hline & & $\begin{array}{l}\text { V. A.med } \\
\text { g/s }\end{array}$ & & & & +++ & +++ & & ++ & +++ \\
\hline
\end{tabular}




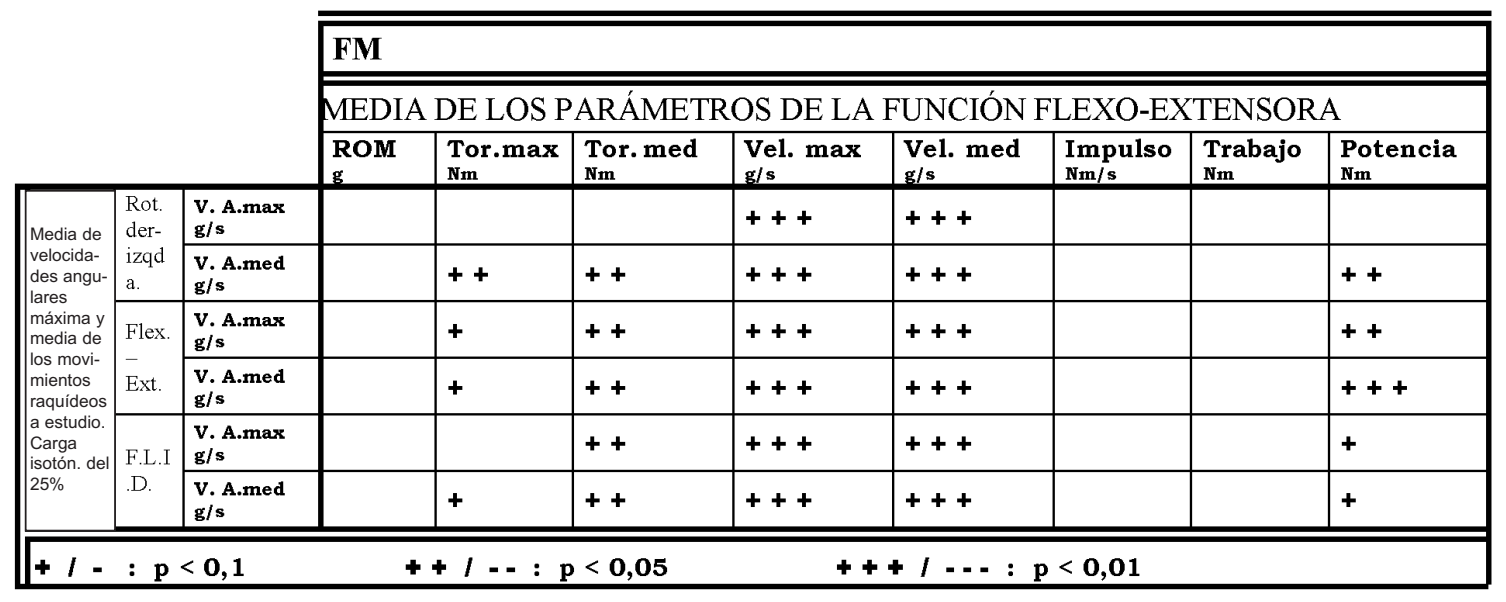

\begin{tabular}{|c|c|c|c|c|c|c|c|c|c|}
\hline & \multicolumn{6}{|c|}{ REFERENCIA } \\
\hline & & & & \multicolumn{6}{|c|}{$\begin{array}{l}\text { MEDIA DE VELOCIDADES ANGULARES MAXIMA Y MEDIA DE LOS } \\
\text { MOVIMIENTOS RAQUIDEOS A ESTUDIO CON CARGA ISOTÓNICA DEL } 25 \%\end{array}$} \\
\hline & & & & \multicolumn{2}{|c|}{ Rot. der-izqda. } & \multicolumn{2}{|c|}{ Flex. - Ext. } & \multicolumn{2}{|c|}{$\begin{array}{l}\text { F.L.I.D. } \\
\end{array}$} \\
\hline & & & & $\begin{array}{l}\text { V. A. } \max \\
\mathrm{g} / \mathrm{s}\end{array}$ & $\underset{\mathrm{g} / \mathrm{s}}{\text { V. A. med }}$ & $\underset{\mathrm{g} / \mathrm{s}}{\text { V. A. } \max }$ & $\underset{\mathrm{g} / \mathrm{s}}{\text { V. A. med }}$ & $\begin{array}{l}\text { V. A. } \max \\
\mathrm{g} / \mathrm{s}\end{array}$ & $\underset{\mathrm{g} / \mathrm{s}}{\text { V. }}$. med \\
\hline \multirow{7}{*}{$\begin{array}{l}\mathbf{F} \\
\mathbf{M}\end{array}$} & \multirow{6}{*}{\begin{tabular}{||l} 
Media de \\
velocida- \\
des \\
angula- \\
res máxi- \\
ma y \\
media de \\
los movi- \\
mientos \\
raquí- \\
deos a \\
estudio. \\
Carga \\
isotónica \\
del $25 \%$
\end{tabular}} & \multirow{2}{*}{$\begin{array}{c}\text { Rot. } \\
\text { der- } \\
\text { izqda }\end{array}$} & $\begin{array}{c}\text { V. A. } \\
\max \\
g / \mathbf{s}\end{array}$ & & + & + & & +++ & \\
\hline & & & $\begin{array}{c}\text { v. A. } \\
\text { med } \\
\text { g/s }\end{array}$ & +++ & & + & & ++ & +++ \\
\hline & & \multirow{2}{*}{$\begin{array}{c}\text { Flex. } \\
- \\
\text { Ext. }\end{array}$} & $\begin{array}{c}\text { V. A. } \\
\max \\
\text { g/s }\end{array}$ & +++ & +++ & & & ++ & +++ \\
\hline & & & $\begin{array}{c}\text { v. A. } \\
\text { med } \\
\text { g/s }\end{array}$ & +++ & +++ & +++ & & & \\
\hline & & \multirow{2}{*}{$\begin{array}{l}\text { F.L.I: } \\
\text { D. }\end{array}$} & $\begin{array}{l}\text { V.A. } \\
\max \\
\text { g/s }\end{array}$ & +++ & +++ & +++ & +++ & & ++ \\
\hline & & & $\begin{array}{c}\text { V.A. } \\
\text { med } \\
\text { g/s }\end{array}$ & +++ & +++ & +++ & +++ & +++ & \\
\hline & \multicolumn{4}{|c|}{$+I-: \mathrm{p}<0,1$} & $++1=$ & $\mathrm{p}<0,05$ & \multicolumn{3}{|c|}{$+++I \ldots: p<0,01$} \\
\hline \multicolumn{10}{|c|}{$\begin{array}{l}\text { Presentación del análisis multiparamétrico sobre los resultados obtenidos con los distintos } \\
\text { parámetros isométricos medidos buscando la existencia de correlación de Pearson en la muestra } \\
\text { poblacional de referencia y en la muestra poblacional con sindrome de Fibromialgia en su conjunto } \\
\text { (FM). Se describen en cada cuadrícula la probabilidad, con su signo, de que los resultados se ajusten } \\
\text { considerando tres niveles : muy probable }(p<0,01) \text {, probable }(p<0,05) \text {, probabilidad incierta }(\mathrm{p} \\
<0,1) \text {. Alguno de los campos se han dividido en dos áreas independientes mediante una diagonal de } \\
\text { cuadriculas rayadas, para representar en la misma tabla los resultados de las sendas muestras } \\
\text { poblacionales. Las cuadriculas en blanco indican que la probalilidad de correlación era }>/=0,1 \text {. E1 } \\
\text { nombre completo de cada indicador y su abreviatura esta detallado en la Tabla } 1 \text {. }\end{array}$} \\
\hline
\end{tabular}

De estas tablas se pueden extraer una serie de relaciones entre parámetros, todas ellas de signo positivo, que se describen a continuación diferenciandolas según el grupo analizado.

\section{Correlaciones evidentes en la muestra poblacional de referencia:}

- En el capítulo de capacidades isométricas entre Flex., Ext., F.L.D. y F.L.I..
- En el capítulo media de los parámetros de la función flexo-extensora:

- Entre Tor.max, Tor.med e impulso.

- Entre Vel.max, trabajo y potencia.

- En el capítulo media de las velocidades angulares máxima y media de los movimientos raquídeos a estudio con carga isotónica del $25 \%$.

- Entre V.A.max/Rot.der-izqda, V.A. med/ Rot.der-izqda y V.A.max/F.L.I.D. 
Indicadores bioquímicos y pruebas isométricas en fibromialgia Manuel López Espino, Adolfo Gozalo Palomares, José Carlos Mingote Adán, César Borobia Fernández

- Entre V.A.med/Rot.der-izqda y V.A. med / F.L.I.D.

- Entre V.A.max/F.L.I.D, V.A.max/ F.L.I.D y V.A.max/Flex-Ext.

- Entre los capítulos de movi ROM y media de los parámetros de la función flexo-extensora en rotaciones y Tor max/Tor med.
- Entre los capítulos media de los parámetros de la función flexo-extensora y media de las velocidades angulares máxima y media de los movimientos raquídeos a estudio con carga isotónica del $25 \%$ :

- Vel.max/Vel.med de la función flexo extensora y V.A.max/Flex-ext y V.A. $\max$ y V.A.med/F.L.I.D.

Cuadro 3. Interrelación entre indicadores bioqímicos de estrés y parámetros isométricos

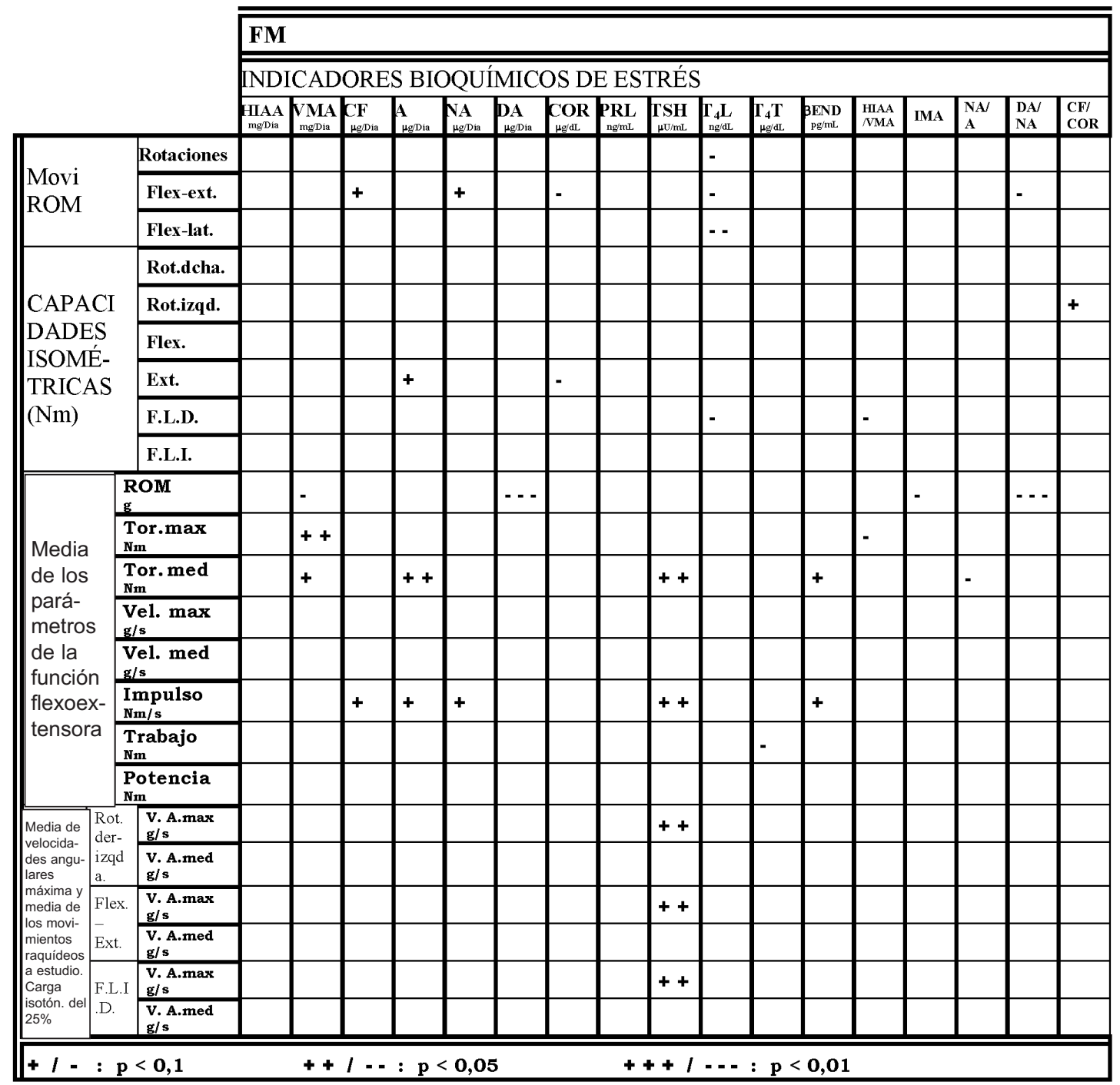

\footnotetext{
Presentación del análisis multiparamétrico sobre los resultados obtenidos con los distintos indicadores bioquímicos de estrés y los parámetros isométricos medidos, buscando la existencia de correlación de Pearson en la muestra poblacional con sindrome de Fibromialgia en su conjunto (FM). Se describen en cada cuadricula la probabilidad, con su signo, de que los resultados se ajusten considerando tres niveles : muy probable ( $\mathrm{p}<$ $0,01)$, probable $(p<0,05)$, probabilidad incierta $(p<0,1)$. Las cuadriculas en blanco indican que la probalilidad de correlación era $>/=0,1$. El nombre completo de cada indicador y su abreviatura esta detallado en la Tabla 1.
} 
- Trabajo/Potencia de la función flexo extensora y V.A.max/Flex-ext y V.A. max y V.A.med/F.L.I.D.

\section{Correlaciones evidentes en la muestra de pacientes con fibromialgia:}

- En los capítulos de movi ROM y capacidades isométricas se correlacionan casi todos los parámetros entre sí con excepción de Rot.dcha (capacidades isométricas) y las medidas de movi ROM.

- Entre los capítulos de movi ROM y media de los parámetros de la función flexo-extensora entre Flex-ext (movi ROM) y ROM, Vel.max, Vel.med y potencia (función flexo-extensora).

- Entre los capítulos de capacidades isométricas y la media de los parámetros de la función flexo-extensora se centran más en Tor.max/ Tor.med y Trabajo/Potencia.

- Entre los capítulos de movi ROM y la media de las velocidades angulares máxima y media de los movimientos raquídeos a estudio con carga isotónica del $25 \%$ destacan los de la función flexo-extensora.

- Entre los capítulos de capacidades isométricas y la media de las velocidades angulares máxima y media de los movimientos raquídeos a estudio con carga isotónica del $25 \%$ existen en rot.izq/flex y F.L.D..

- Entre los capítulos de media de los parámetros de la función flexo-extensora y la media de las velocidades angulares máxima y media de los movimientos raquídeos a estudio con carga isotónica del $25 \%$ en Vel.max/Vel.med, potencia $\mathrm{y}$, aunque menos evidente en tor.med/ tor.max.

Teniendo en cuenta que una disfunción orgánica puede tener reflejo metabólico-funcional hemos confrontado los datos bioquímicos con los isométricos con el siguiente resultado. Cuadro 3.

\section{DISCUSION Y CONCLUSIONES}

La fibromialgia no es un trastorno mental, aunque se haya mal interpretado como una depresión enmascarada, sino que se trata de una enfermedad orgánica como el síndrome de fatiga crónica y el colon irritable, parcialmente condicionadas por diferentes alteraciones psicopatológicas. Diferentes situaciones estresantes pueden influir en el desencadenamiento y en el curso de la enfermedad, como un impacto negativo sobre la misma.
La fibromialgia no es una enfermedad reumatológica, sino que constituye el prototipo de enfermedad psicosomática compleja que incluye una alteración principal en el sistema nervioso central con afectacuión del sistema nociceptivo que es hiperreactivo a estímulos no solo dolorosos sino también a estímulos estresantes y que llega a hiperactivarse de forma permanente, proceso denominado como desensibilización central permanente, recientemente objetivada por técnicas de neuroimagen como resonancia magnética espectroscópicas.(32)

La fibromialgia se ha clasificado provisionalmente dentro de los denominados síndromes somáticos funcionales, por la ausencia de hallazgos exploratorios que expliquen estos padecimientos, a diferencia de las enfermedades psicosomáticas, que son entidades médicas genéticamente condicionadas y relacionadas con una respuesta patológica al estrés crónico.

\section{RESULTADOS}

Estas investigaciones realizadas tienen por objeto avanzar en el conocimiento de las alteraciones neurobiológicas que constituyen el proceso fisiopatológico central responsable del síndrome fibromiálgico, así como para avanzar en la objetivación diagnóstica de esta supuesta "enfermedad invisible" y en su tratamiento eficaz. Podría tratarse de un trastorno psicosomático complejo que incluye un intenso dolor generalizado crónico de origen central (hiperalgesia y alodimia) por procesamiento nociceptivo anormal (sensibilización central o kindling, sumación temporal, etc.) disregulación neuroendocrina y del ritmo vigilia-sueño, déficit cognitivos significativos como los de concentración-atención, memoria de trabajo y varias disfunciones ejecutivas así como otros frecuentes síntomas y síndromes psicosomáticos como el colon irritable y la jaqueca.

No obstante, al tratarse de estudios transversales no es posible demostrar una causalidad directa entre dolor crónico, alteraciones psicopatológicas tales como depresión, ansiedad y el comportamiento de quienes los padecen. El predominio de conductas de riesgo (sedentarismo, tabaquismo, etc.) sobre los buenos hábitos de salud contribuyen a la cronificación y al empeoramiento clínico de los pacientes. Un significativo porcentaje de pacientes con fibromialgia, entre un $25-30 \%$ no responden al tratamiento y padecen un importante grado de incapacidad general y de tipo laboral, aunque tienen muchas dificultades para lograr percibir las justas y necesarias prestaciones económicas de la seguridad social, a pesar, de padecer un alto 
grado de deterioro físico y mental, según revisan Wolfe y Potter (30) Estos estudios permiten identificar biomarcadores diagnósticos que legitiman la realidad de la demanda de estas pacientes y mejoran la validez diagnóstica de esta entidad clínica.

\section{CONCLUSIONES}

1. Debemos partir del hecho de que existen numerosas disfunciones orgánicas que no siempre se reflejan en alteraciones bioquímicas y fisiopatológicas específicas por lo que no es sencillo extraer conclusiones definitivas, aún así convendría apuntar en esta tabla comparativa que las pacientes con fribomialgia presentan:

2. Correlación negativa entr T4L y movi ROM.

3. Correlación positiva entre TSH y Tor.med e impulso (media de los parámetros de la función flexo-extensora) y V:A:max en rotaciones, flex-ext y

\section{BIBLIOGRAFIA}

1. Wolfe F, Smythe HA, Yunus MB, Bennett RM, Bombandier C, Goldenberg DL. The American College of Rheumathology 1990 Criteria for the Clasification of Fibromyalgia. report of the Multicenter Criteria Committee. Arthritis Rheum 1990; 33: $160-72$.

2. Myopain '92. Abstracts from the $2^{\text {nd }}$ World Congress on Myofascial Pain and Fibromyalgia. Copenhagen, Denmark, August 17-20, I.

3. Quintner J. Fibromyalgia: The Copenhagen Declaration. Lancet 1992 oct 31; 340 (8827): 1103.

4. Wolfe Frederick, and The Vancouver FM Consensus Group Special report. The Fibromyalgia syndrome: A consensus report on Fibromylagia and Disability. J Rheumatol 1996; 23: 534-539.

5. Yunus MB, Khan MA, Karolyn $\mathrm{K}$ and Col. Genetic linkage in the fibromialgia syndrome. Journal of Henmatology 1999, 26: 408-412

6. Simms RW, Goldenberg DL, Felson DT, et al: Tenderness in 75 anatomic sites: distinguishing fibromyalgia patients from control. Arthritis Rheum, 1988; 31: 182-187.
F.L.I.D. (media de las velocidades angulares máxima y media de los movimientos raquídeos a estudio con carga isotónica del $25 \%$ ).

4. Correlación negativa entre DA y ROM (media de los parámetros de la función flexo-extensora).

5. Correlación positiva entre VMA y Tor.max (media de los parámetros de la función flexo-extensora).

6. Correlación positiva entre A y Tor.med (media de los parámetros de la función flexo-extensora).

Al tratarse de una patología neurobiológica compleja requiere de un abordaje multidisciplinar, realizado según el modelo de la medicina psicosomática, en el que es fundamental incluir especialistas de los servicios de neurología, de las unidades del dolor, rehabilitadotes y fisioterapeutas, psicólogos y psiquiatras, además de reumatólogos.

7. Mera Varela AJ: Fibrositis (fibromialgia) primaria. Inflamación, 93, 1991; 2(4): 265-268.

8. Mountz JM, Bradley LA, Modell JG, Alexander RW, Triana-Alexander M, Aaron LA, et al: Fibromyalgia in women. Abnormalities of regional cerebral blood flow in talamus and the caudate nucleus are associated with low pain threshold levels. Arthritis Rheum, 1995; 38 (7): 926-938.

9. Síndrome fibromiálgico. En: Compendio de las enfermedades reumáticas. Ed Salvat. 1994; 8284.

10. Moldofsky H, Warsh JJ. Plasma tryptophan and musculoskeletal pain in non-articular rheumatism ("fibrositis syndrome"). Pain1978 Jun; 5 (1): 65-71.

11. Russell IJ: Neurohormonal aspects of fibromyalgia syndrome. Rheum Dis Clin North Am, 1989; 15: 149-167.

12. Moldofsky H: Sleep and fibrositis syndrome. Rheum Dis Clin North Am, 1989; 15: 90-103.

13. Smithe HA, moldofsky H: Two contributions to understanding the fibrositis syndrome. Bull Rheum Dis, 1977-78; 28:928-931. 
14. Moldofsky H., Scarisbrick P., England R., Smythe H.: Musculoskeletal symptoms and NonRem sleep disturbance in patients with "Fibrositis Syndrome" and healthy subjets. Psychosomatic Medicine, 1975; 37(4): 341-351.

15. Moldofsky H. And Scarisbrick P.: Induction of neurasthenic musculoskeletal pain syndrome by selective sleep stage deprivation. Phychosomatic Medicine, 1976; 38(1): 35-44.

16. Moldofsky H. And Lue F. A.: The relationship of alpha and delta EEG frequences to pain and mood in "fibrositis" patients treated with chlorpromazine and L-tryptophan. Electroencephalography and clinical neurophysiology, 1980; 50: 71-80.

17. Bennet RM. Adult growth hormone deficiency in patients with fibromyalgia. Curr Rheumatol Rep aug 2002; 4, 4: 306-12.

18. Povedano Gómez J.B, Iglesia Salgado J. L. Fibromialgia: Tema monográfico. Rev Esp Reumatol 2000; 27: 426-429.

19. Klein R, Bansch M, Berg PA. Clinical relevance of antibodies against serotonin and gangliosides in patients with primary fibromyalgia syndrome. Psychoneuroendocrinology 1992 Nov; 17(6): 593-8.

20. Werle E, Fischer HP, Muller A, Fiehn W, Eich W. Antibodies against serotonin have no diagnotic relevance in patients with fibromyalgia syndrome. J. Rheumatol 2001 Mar; 28 (3): 595-600.

21. Aarflot T, Buusgaard D. Association between chronic widepread musculosketal complaints and thyroid autoinmunity. Results from a community survey. Scand J Prim Health Care jun 1996; 14, 2:111-5.

22. Van West D, Maes M. Neuroendocrine and immune aspects of fibromyalgia. BioDrugs 2001; 15, 8: 521-31.
23. Lund N, Bengtsson A, Thorborg P. Muscle tissue oxygen pressure primary fibromyalgia. Scand J Rheumatol 1986; 15: 165-73.

24. Francisco Javier Cano y Luis Rodríguez: Evaluación del lenguaje interno ansiógeno y depresógeno en la experiencia de dolor crónico. Apuntes de psicología, 2002, Vol. 20, num 3, pp. 329-346.

25. Neeck G, Riedel W. Thyroid function in patients with fibromyalgia syndrome. J Rheumatol 1992 Jul;19(7):1120-2.

26. Bennett RM: Muscle physiology and cold reactivity in the fibromyalgia syndrome. Rheum Dic Clin North Am, 1989; 15:135-147.

27. Awad EA: Intestinal myofibrositis: hipothesis of the mechanism. Arch Phys Med Rehabil, 1973; 49: 155.

28. Mingote, Jose Carlos y Arigita, Ivón. Jornadas de la mujer. Instituto de la mujer. 2000.

29. De Bléclourt AC, Wolf RF, Van Rijswijk MH, Kamman RL, Knipping AA, Mooyart EL. In vivo 31P Magnetic Resonance Spectroscopy (MRS) of tender points in patiens with primary fibromyalgia syndrome. Rheumatol Int 1991; 11: 51-54.

30. Idoate Garcia VM, Álvarez Erviti s. y col. Clasificación de las fibromialgias según la capacidad funcional medida con Isostation B-200. Medicina y seguridad del trabajo, (15-20), No 191, 2002

31. Wolfe F. y potter j.: Fibromyalgia and work disability. Controversies in fibromyalgia and related conditions. 1996

32. Collado A. Sistema Tálamo-prefrontal en la fibromialgia.Congreso Nacional de Psiquiatría. Valencia 2008. 